\title{
Integration of phylogenomics and molecular modeling reveals lineage-specific diversification of toxins in scorpions
}

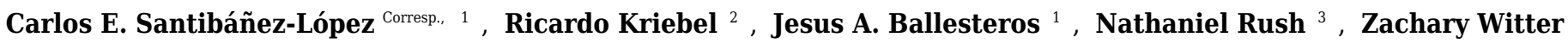

${ }^{3}$, John Williams ${ }^{3}$, Daniel A. Janies ${ }^{3}$, Prashant P. Sharma ${ }^{\text {Corresp. } 1}$

1 Department of Integrative Biology, University of Wisconsin-Madison, Madison, Wisconsin, United States

2 Department of Botany, University of Wisconsin-Madison, Madison, Wisconsin, United States

3 Department of Bioinformatics and Genomics, University of North Carolina at Charlotte, Charlotte, North Carolina, United States

Corresponding Authors: Carlos E. Santibáñez-López, Prashant P. Sharma

Email address: santibanezlo@wisc.edu, prashant.sharma@wisc.edu

Scorpions have evolved a variety of toxins with a plethora of biological targets, but characterizing their evolution has been limited by the lack of a comprehensive phylogenetic hypothesis of scorpion relationships grounded in modern, genome-scale datasets. Disagreements over scorpion higher-level systematics have also incurred challenges to previous interpretations of venom families as ancestral or derived. To redress these gaps, we assessed the phylogenomic relationships of scorpions using the most comprehensive taxonomic sampling to date. We surveyed genomic resources for the incidence of calcins (a type of calcium channel toxin), which were previously known only from 16 scorpion species. Here, we show that calcins are diverse, but phylogenetically restricted only to parvorder lurida, one of the two basal branches of scorpions. The other branch of scorpions, Buthida, bear the related LKTx toxins (absent in lurida), but lack calcins entirely. Analysis of sequences and molecular models demonstrates remarkable phylogenetic inertia within both calcins and LKTx genes. These results provide the first synapomorphies (shared derived traits) for the recently redefined clades Buthida and lurida, constituting the only known case of such traits defined from the morphology of molecules. 
1 Integration of phylogenomics and molecular modeling reveals lineage-specific diversification of

2 toxins in scorpions

3

4 Carlos E. Santibáñez-López, ${ }^{* 1}$ Ricardo Kriebel, ${ }^{2}$ Jesús A. Ballesteros ${ }^{1}$, Nathaniel Rush, ${ }^{3}$ Zachary Witter, ${ }^{3}$

5 John Williams, ${ }^{3}$ Daniel Janies ${ }^{3}$ and Prashant P. Sharma ${ }^{* 1}$

$6{ }^{1}$ Department of Integrative Biology, University of Wisconsin-Madison, 430 Lincoln Drive, Madison, WI 7 53706, USA.

$8{ }^{2}$ Department of Botany, University of Wisconsin-Madison, 430 Lincoln Drive, Madison, WI 53706, 9 USA.

$10{ }^{3}$ Department of Bioinformatics and Genomics, University of North Carolina at Charlotte, Charlotte, NC, 11 28223, USA.

12

13 *Correspondence: CESL (santibanezlo@wisc.edu), PPS (prashant.sharma@wisc.edu). 14

15

16 


\section{Abstract}

18 Scorpions have evolved a variety of toxins with a plethora of biological targets, but characterizing their evolution has been limited by the lack of a comprehensive phylogenetic hypothesis of scorpion relationships grounded in modern, genome-scale datasets. Disagreements over scorpion higher-level systematics have also incurred challenges to previous interpretations of venom families as ancestral or

22 derived. To redress these gaps, we assessed the phylogenomic relationships of scorpions using the most 23 comprehensive taxonomic sampling to date. We surveyed genomic resources for the incidence of calcins 24 (a type of calcium channel toxin), which were previously known only from 16 scorpion species. Here, we 25 show that calcins are diverse, but phylogenetically restricted only to parvorder Iurida, one of the two basal 26 branches of scorpions. The other branch of scorpions, Buthida, bear the related LKTx toxins (absent in 27 Iurida), but lack calcins entirely. Analysis of sequences and molecular models demonstrates remarkable phylogenetic inertia within both calcins and LKTx genes. These results provide the first synapomorphies (shared derived traits) for the recently redefined clades Buthida and Iurida, constituting the only known case of such traits defined from the morphology of molecules.

\section{Introduction}

Scorpions are an iconic group of arachnids that are central to investigations of arthropod terrestrialization, morphological stasis, and diversification of body plans (Kjellesvig-Waering, 1986; Jeram, 2005; Sharma et al., 2014; Waddington, Rudkin \& Dunlop, 2015). To scientists and laypersons, scorpions are particularly fascinating for the diversity of their venom, a complex mixture of bioactive compounds (e.g. peptides, proteins) secreted in specialized organs and used to disrupt biochemical and physiological processes in target organisms (King \& Hardy, 2013; Casewell et al., 2013; Haney et al., 2016). Among scorpions, venoms are rich in toxins with a broad array of biological targets, including those affecting $\mathrm{Na}^{+}, \mathrm{K}^{+}, \mathrm{Cl}^{-}$and $\mathrm{Ca}^{2+}$ ion channels (Possani et al., 1999; Sunagar et al., 2013; Santibáñez-López \& 42 Possani, 2015).

The origin of toxins in animal venom has been inferred to be the result of recruitment of paralogs of ancestral housekeeping genes, followed by diversification and neofunctionalization, a process driven by positive selection (Juarez et al., 2008; Fry et al., 2009; Rokyta et al., 2011; Wong \& Belov, 2012; Haney et al., 2016; Dowell et al., 2016). While novel peptides often preserve the same molecular scaffold of their ancestral protein, key changes in functional residues, mostly in surface-exposed sites, acquire newly derived biological activities (Fry et al., 2009; Casewell et al., 2013). Nevertheless, two peptides with statistically insignificant sequence similarity can also adopt the same scaffold (Orengo, Jones \& 
51 Thornton, 1994), resulting in evolutionary convergence in fold structures, and thus rendering inference of

52 homology non-trivial.

53

54 The study of scorpion venom diversity is further complicated by the patchiness of existing taxonomic

55 sampling. The advent of current-generation sequencing technology has greatly advanced the discovery of

56 venom diversity through the availability of transcriptomes and the first scorpion genomes. However, data

57 acquisition strategies asymmetrically favor the taxonomic sampling of Buthidae, the largest of the 20

58 described scorpion families (of the approximately 2400 described species of scorpions, $48 \%$ are buthids).

59 Buthidae are also intensely sampled because this family contains nearly all scorpion species of medical

60 significance. Comparatively fewer resources exist for the remaining 19 scorpion families, and these have

61 revealed additional molecular diversity that is not reflected in Buthidae (Santibáñez-López et al., 2016;

62 2017).

63

64 One such example of this diversity are calcins (ryanodine receptor ligands), a group of inhibitor cystine knot (ICK)-stabilized peptides found in scorpion venom. Calcins rapidly activate ryanodine receptors (RyRs) in cardiac or skeletal muscle cells in mammals with high affinity and specificity (reviewed in Xiao et al (Xiao et al., 2016)). These peptides bind to cell surface glycosaminoglycans and membrane lipids (Mabrouk et al., 2007; Ram et al., 2008), translocating also into cells, and undergo posttranslational modifications by target cell enzymes (Ronjat et al., 2016). To date, calcins have only been discovered in 16 species from eight families (but not Buthidae). Intriguingly, calcins share phylogenetic affinities with the lambda potassium channel toxins (hereafter "LKTx") (Gao et al., 2013; Santibáñez-López et al., 2016) only found in species of Buthidae. LKTx inhibits the $\mathrm{K}^{+}$channel in insects without the inactivation of the skeletal-type $\mathrm{Ca}^{+2}$ ryanodine receptors in mammals. It is therefore unknown when calcins evolved in the scorpion tree of life; limitations in sampling preclude inference of whether calcins are prone to evolutionary loss and/or replacement by other toxins in such lineages as buthids.

A separate impediment to analysis of venom evolution within a phylogenetic context is a series of recent changes in understanding of scorpion basal relationships, precipitated by the advent of phylogenomic datasets (Soleglad \& Fet, 2003; Coddington et al., 2004; Sharma et al., 2015). The phylogenomic tree of Sharma et al. (2015) fundamentally changed the systematics of the two basal branches of the scorpion tree

81 of life, suggesting that the sister groups of Buthidae were the relictual south-east Asian families

82 Chaerilidae and Pseudochactidae (which is also found in parts of central Asia). This work was 83 nevertheless limited to 25 species and focused on a different subset of taxa from the one wherein calcins 84 and LKTx peptides have been reported. A recent approach using a different molecular dataset 
85 (ultraconserved elements) also recovered further differences in derived parts of the tree topology, but

86 were limited to six scorpion species, and did not include either Chaerilidae and Pseudochactidae (Starrett

87 et al., 2017). The present ambiguity of scorpion basal relationships thus hinders reconstruction of venom

88 evolution.

89

90 To achieve a comprehensive, integrated understanding of scorpion phylogeny and calcin/LKTx evolution,

91 we inferred scorpion relationships using the most comprehensive phylogenomic dataset to date,

92 maximizing the overlap between phylogenetically distinctive lineages and existing datapoints for ICK

93 peptides. We separately surveyed venom gland transcriptomes to discover and map the distribution of

94 ICK homologs. To assess the evolutionary dynamics of calcins and LkTx peptides, we inferred three-

95 dimensional molecular models and performed parametric comparative analyses of protein folding and

96 biochemical properties. Here we show that calcins and LKTx are reciprocally restricted to the two most

97 basally branching clades of scorpions and exhibit marked phylogenetic inertia in protein shape. The

98 identification of LKTx in venom gland transcriptomes of Buthidae, Chaerilidae, and Pseudochactidae

99 validates the monophyly of this group and constitutes the first molecular synapomorphy uniting this

100 parvorder, Buthida.

101

102 Materials and Methods

103

104 Taxon sampling and orthology inference

105

106 We assembled a dataset of 55 scorpion species and 13 chelicerate outgroups, consisting of one complete

107 scorpion genome, 13 EST libraries, six 454-pyrosequencing transcriptomes, and 49 Illumina

108 transcriptomes (two newly generated for this study and 44 previous libraries generated by our research

109 group; Table S1). Specimens of Kolotl magnus and Urodacus elongatus were dissected into RNAlater

110 solution (Ambion). Total RNA was extracted using the Trizol Trireagent system (Ambion Life

111 Technologies, Waltham, MA, USA). Libraries were constructed in the Apollo 324 automated system

112 using the PrepX mRNA kit (IntegenX), with samples marked with unique indices to enable multiplexing.

113 Concentration of the cDNA libraries was measured using the dsDNA High Sensitivity (HS) assay in a

114 Qubit v 2 fluorometer (Invitrogen). Library quality and size selection were checked using an Agilent 2100

115 Bioanalyzer (Agilent Technologies) with the HS DNA assay. The samples were run using the Illumina

116 HiSeq 2500 platform with paired-end reads of 100 or $150 \mathrm{bp}$ at the FAS Center for Systems Biology at

117 Harvard University. De novo assemblies were conducted using Trinity v 2.8, keeping a path

118 reinforcement distance parameter of 75 (Grabherr et al., 2011). The search for orthologous sequences to 
119 infer species trees was also conducted de novo using a phylogenetically informed orthology criterion, as

120 implemented in UPhO v 1.0 (Ballesteros \& Hormiga, 2016). The sequences of four representative species,

121 Urodacus planimanus, Anuroctonus australis, Mesobuthus martensii and Chaerilus celebensis were

122 combined, and used as query against the database containing all species in this study using the option

123 blastp. The representative species strategy was preferred over "all versus all" searches to avoid the

124 computational burden imposed by exhaustive pairwise sequence comparisons. Sequences were clustered

125 in gene families using $\mathrm{mcl}$ (Dongen, 2000; Enright, Van Dongen \& Ouzounis, 2002). A variety of values

126 for the inflation parameter were explored $i=\{1.4,2,4,6\}$ and the clustering produced with $i=6$ was

127 selected from the alternative clustering based on the efficiency scores reported by $\mathrm{mcl}$. A total of 9,930

128 clusters produced with at least 20 species was carried for downstream analyses.

129

130 Gene-family trees (GFTs) were estimated for each cluster using FastTree v 2 (Price, Dehal \& Arkin,

131 2010) following multiple sequence alignment with MAFFT v 7.0 with the parameters -anysymbol and -

132 auto (Katoh \& Standley, 2013). Masking of ambiguously aligned regions was performed using trimAl

133 v1.2 with the -gappyout algorithm (Capella-Gutiérrez, Silla-Martínez \& Gabaldón, 2009) and removing

134 sequences that after trimming had less than 50 amino acids or less than $25 \%$ unambiguously aligned sites.

135 Parallelization of the phylogenetic pipeline was implemented through gnu-parallel (Tange, 2011). The

136 resulting gene family trees were analyzed in search of groups of orthologs with at least 19 different

137 species using UPhO (orthology inference parameters: -m 19 -S 0.75 -iP; Ballesteros \& Hormiga, 2016),

138 resulting in 3110 orthologs. The individual orthogroup alignments were concatenated in a supermatrix

139 with geneStitcher.py (Ballesteros \& Hormiga, 2016). In-paralogs, alleles, duplicates and/or splice-variants

140 retained in the orthologs were resolved in favor of the longest sequence. The resulting matrix (henceforth,

141 Matrix 1), consisted of 3110 loci, 792210 aligned amino acid sites, and 69\% missing data.

142

143

Phylogenomic inference and molecular dating

144

145

Maximum likelihood (ML) analyses were performed using ExaML v 3.0 (Kozlov, Aberer \& Stamatakis,

146 2015) and IQ-TREE v 1.5.5 (Nguyen et al., 2014), implementing ultrafast bootstrap resampling to gauge

147 nodal support (Minh, Nguyen \& Haeseler, 2013). The matrix was partitioned by locus, selecting the best-

148 fitting amino acid substitution model per partition (based on the automatic model assignment obtained

149 during the ExaML run with the default ML criterion) and with per-site rates (ExaML) or with four gamma

150 categories (in IQ-TREE). The resulting maximum likelihood tree was calibrated for downstream analyses

151 using penalized likelihood (Sanderson, 2002) as implemented in the chronos function of the R package

152 ape (Paradis, Claude \& Strimmer, 2004; Paradis, 2013), under the relaxed and correlated models and a 
153 family of values for lambda $=\{0.5,1.0\}$. Age constraint strategy follows the calibration point previously

154 used and justified in our recent works (Santibáñez-López, Kriebel \& Sharma, 2017; Sharma et al., 2018),

155 setting the lower bound of the crown age of Opiliones to $411 \mathrm{Myr}$; the crown age of Araneae to $305 \mathrm{My}$;

156 the stem age of Amblypygi to $385 \mathrm{Myr}$ and the stem age of Buthidae to a minimum of $120 \mathrm{Ma}$ (see

157 Sharma et al., 2018). Alternative dating strategies (e.g. Bayesian inference with explicit clock models)

158 were explored previously in our recent work on scorpion basal diversification, and yielded congruent

159 results with penalized likelihood using the complete matrix (ref. Sharma et al., 2018).

160

161 To explore the trade-off between matrix size and matrix completeness, three additional matrices were

162 constructed by varying the inclusion threshold for minimum number of species present per locus. To

163 maximize informativeness in the concatenated analysis, we treated our original matrix (Matrix 1, above)

164 with the Matrix Reduction (MARE) algorithm, which eliminates uninformative genes and taxa, using

165 default parameters (Meusemann et al., 2010). The resulting dataset was termed Matrix 2 and consisted of

16643 terminals and 1632 genes (30\% missing data). The search of groups of orthologs was conducted using

167 a different number of species (parameter $-\mathrm{m}$ in $\mathrm{UPhO}$ ). An intermediately stringent matrix required the

168 orthologs to be present in at least 35 species (Matrix 3; 66 terminals; 799 genes; 39\% missing data).

169 Lastly, the most stringent matrix required orthologs to be present in at least 45 species (Matrix 4; 52

170 terminals, 108 genes; $13 \%$ missing data).

171

172 As concatenation methods can mask phylogenetic conflict when strong gene tree incongruence is

173 incident, we conducted species tree estimation of the constituent orthologs of the four matrices mentioned

174 above, and an additional matrix, which required only five terminals to be present per locus (Matrix 5;

17525997 genes) using the gene trees generated with FastTree2 (as mentioned above) and ASTRAL-II

176 (Mirarab \& Warnow, 2015). While molecular dating analyses were explored for Matrix 2 (selected as a

177 compromise between higher number of loci and lower values of missing data) using PhyloBayes-mpi v

1781.5 (Lartillot et al., 2013) under a CAT + GTR + $\Gamma$ model, analyses failed to converge after over two

179 months of computation time on four independent runs in eight processors; this analysis was excluded

180 from the study.

181

182 Gene tree analysis of ICK peptides

183

184 ICK homologs were retrieved from the complete dataset used in the scorpion phylogenetic analyses, as

185 well as from GenBank and UniProt (Table S2). The signal peptides, propeptides and mature peptides

186 were predicted using SpiderP from Arachnoserver (Herzig et al., 2010). Outgroup taxa for gene tree 
187 analysis consisted of three spider calcium channel toxins (ICK peptides); to root the tree, two disulfide-

188 directed $\beta$-hairpin (DDH) scorpion toxins were selected: Phi-liotoxin isolated from the venom of

189 Liocheles waigiensis (Smith et al., 2013), and the DDH-Uro-1 deduced from a cDNA cloned from

190 Urodacus manicatus (Sunagar et al., 2013). The phylogenetic relationship of scorpion DDH and ICK

191 peptides has been discussed elsewhere (Smith et al., 2013; Sunagar et al., 2013). Multiple sequence

192 alignments for the full precursor were generated using MAFFT v 7.0 (Katoh \& Standley, 2013), resulting

193 in a matrix consisting of 64 terminals and 132 amino acid sites. Bayesian inference (BI) analysis was

194 performed with MrBayes v 3.2.2 (Ronquist et al., 2012) using the Dayhoff model selected under the

195 Bayesian information criterion, as selected by ProtTest v 3 (Darriba et al., 2011). Four runs, each with

196 four Markov chains were implemented for $1 \times 10^{7}$ generations using default priors and discarding $1 \times 10^{6}$

197 generations using default priors.

198

199

Molecular models

200

201 Multiple sequence alignments of the mature peptide nucleotide sequences were generated based on their 202 corresponding mature peptide amino acid sequences using PAL2NAL v 14 (Suyama, Torrents \& Bork,

203 2006). The resulting codon alignment was further used for synonymous and non-synonymous substitution 204 rates using the following models implemented in the datamonkey server (http://datamonkey.org; Weaver 205 et al., 2018): (a) aBSREL (Smith et al., 2015) to test whether a proportion of branches has evolved under 206 positive selection; (b) FUBAR (Murrell et al., 2013) to provide additional support to the detection of sites 207 evolving under positive or negative selection; and (c) MEME (Murrell et al., 2012) to detect episodic or diversifying selection at individual sites in the amino acid sequence. Statistical outcomes under all three approaches are presented in the Supplementary Tables 3-4.

210

211 Three-dimensional structures were generated for 41 calcin mature peptide sequences using the 3D

212 structure of imperacalcin (Lee et al., 2004) in the SWISS-MODEL server (Biasini et al., 2014). The

213 solvent accessible surface area (SAS) for all models was generated using the APBS method (Baker et al., 214 2001) using the PDB2PQR server (Dolinsky et al., 2004). All 3D images were generated and visualized 215 using PyMol v 1.8.2.1. The Accessible Surface Ratio (ASR) was calculated with GetArea (Fraczkiewicz

$216 \&$ Braun, 1998). The molecular weight and volume were calculated based on the pdb files generated using 217 VADAR v 1.8 server (Willard et al., 2003). Other physical and chemical characteristics (e.g. net charge)

218 were calculated based only on the mature peptide sequences using the online peptide calculator

219 (http://www.chinapeptides.com/english/toolcfuben.php) and the ProtParam server

220 (http://web.expasy.org/protparam/) listed in the Supplementary Tables S5-S6. 
221

222

Parametric analyses of molecule shape

223

224 Static images of the frontal and lateral views (as established by Xiao et al., 2016) of the 3D models of all 225 ICK peptides (LKTx and calcins) were generated with PyMol. Files were aligned and exported as png for 226 consistency. Outlines of these frontal and lateral images were extracted from their original images using 227 GIMP v 2.8 (http://www.gimp.org) and converted into monochromatic jpeg files. The geometric 228 morphometric technique of elliptic Fourier analysis (EFA) with the R package Momocs (Bonhomme et 229 al., 2014) was applied to calculate the morphological shape variation of the outlines. Outlines were 230 imported into R, converting them into lists of coordinates as described previously (Santibanez Lopez, 231 Kriebel \& Sharma, 2017). For all structures, 20 harmonics were enough to achieve 99\% of harmonic 232 power during the EFA. The resulting coefficients were summarized using principal components analysis 233 (PCA), which were used to visualize variation in morphospace. These coefficients were also used to 234 calculate mean protein shapes for comparison between groups. For these comparisons, we used the $t p$ iso 235 function in Momocs, which calculates and graphs deformations between two configurations as heatmaps.

236 Specifically, we compared the mean shape between the calcins and LKTxs, as well as the different major 237 clades within calcins.

238

239 In order to place protein morphology in a phylogenetic context, we matched the resulting principal 240 components to the dated molecular phylogeny of scorpions for downstream analyses. This phylogeny was 241 culled to retain the intersection of terminals for which shape data were available at the genus level. This 242 strategy reflects low, or lack of, variation of calcin peptide sequences at the intrageneric level (e.g., the 243 putative calcin sequences of the three species of genus Urodacus were $100 \%$ similar). Visualization of 244 morphospace was conducted using inbuilt functions in Momocs and phytools (Revell, 2012).

245

246 Inference of evolutionary rates

247

248 We investigated the evolutionary dynamics of protein traits by testing for shifts in trait regimes using a 249 continuous multivariate Ornstein-Uhlenbeck (OU) approach implemented in the R library 11ou 250 (Khabbazian et al., 2016). For this analysis, the main axes of shape variation (PC1 and PC2 of the frontal 251 and lateral view from the morphometric analyses) were added to three other characteristics of the 252 proteins: net charge, molecular volume, and molecular weight, for a total of seven columns of data 253 describing the proteins. The data were mapped to the phylogeny and 11ou was used to estimate the best 254 shift configuration and paint the edges of the phylogeny according to their corresponding regime. To 
255 select the best shift configuration, we used the phylogenetic Bayesian Information Criterion (pBIC),

256 which has been shown to be more conservative in assigning regime shifts than the commonly used

257 Akaike Information Criterion (AIC). We assessed statistical support for regime shifts in 11ou with 100

258 bootstrap resampling replicates.

259

260

Scorpion venom database

261

262 To facilitate access to the venom gland transcriptomes that we generated, we established a newly created 263 scorpion venom database online at http://venom.space. Source files for this database consisted of 75

264 inputs. For each scorpion species, an interpro data file, a fasta file, and faa file were created. These files

265 contained headings that were used to establish the structure of the database. After input files were parsed

266 appropriately, they were then used to populate the SQL database and a Django framework was used to

267 create a web application with search functionality. The database includes functionalities for BLAST

268 searches, which users can use to query a specific sequence of interest, and for multiple sequence

269 alignment.

270

271

Results

272

273 Scorpion phylogenomic tree and divergence time estimation

274

275 De novo inference of orthology (i.e., analysis of gene family trees searching for groups of orthologs, with

276 at least 19 different species, with $75 \%$ of support), resulted in the retention of 3110 orthologs. The 3110

277 genes were concatenated into a supermatrix consisting of 792,210 amino acid sites and $69.44 \%$ missing

278 data ("Matrix 1"). The resulting phylogenomic tree supported the reciprocal monophyly of the two basal

279 clades Buthida and Iurida (Figs. 1, S1) with maximal nodal support and stability. Within parvorder

280 Buthida (the families Buthidae, Chaerilidae and Pseudochactidae), a clade comprised of Chaerilidae +

281 Pseudochactidae was recovered as the sister group of Buthidae. Divergence time estimation place the

282 diversification of crown group scorpions between 430-303 Mya (Figs. S2-5). Interestingly, family

283 Hemiscorpiidae was placed as nested in the non-monophyletic Scorpionidae, but with insignificant branch

284 support (Fig. 1, S1).

285

286 Our analyses of additional three supermatrices with different thresholds of gene occupancy (Matrices

$2872,3,4$, see methods) and our species tree analyses with ASTRAL using five different compositional

288 matrices (Matrices 1-5), all recovered similar tree topologies (Fig. S1). 
292 The 59 ICK homologs were represented by 52 scorpion species in 16 families. No ICK peptide sequences 293 were discovered in Liocheles australasiae, Paravaejovis spinigerus, Centruroides (4 spp.), Tityus (3 294 spp.), Isometroides vescus and Lychas buchari. Bayesian inference analyses of a matrix of the 59 ICK 295 homologs (132 amino acid sites) recovered a gene tree subdivided into 41 calcins and 18 LKTx peptides 296 (Fig. 2a). Our results supported some species-specific duplications of a few calcin orthologs, as inferred 297 from clustered pairs of non-identical calcin sequences in the venom of Superstitionia donensis, Brotheas 298 granulatus and Opistophthalmus carinatus. By contrast, the two copies of calcin sequences of Hadogenes 299 troglodytes, plus the two copies of LKTx peptides from Isometrus maculatus, were recovered as out300 paralogs. Oddly, one of the copies of $H$. troglodytes (accession number A0A1B3IJ19) was 100\% identical 301 to the calcin peptide sequence obtained from Scorpiops jendeki (accession number GH548250; Fig. 2a). 302 Comparison of nucleotide sequences of these two calcins revealed only five synonymous changes across 303222 basepairs. To detect the direction of selection acting on the codon sequences of LKTxs and calcins, 304 we used several methods for inferring selection pressure implemented in the server Datamonkey. MEME 305 aims to detect sites evolving under positive selection at a proportion of the branches, but not the entire

306

307

308

309

310

311

312

313 314

315

316

317

318

319

320

321

322

phylogeny, whereas FUBAR assumes the selection pressure for each site is constant across the entire phylogeny. FUBAR found evidence of episodic negative/purifying selection at 4 (LKTx) and 19 (calcin) sites with posterior probability of 0.99 (Fig. 3a,b, Table S3); MEME found one site under the pressure of positive selection in the LKTx sequences, but none in the calcin sequences (Fig. 3c, $d$, Table S4). This same site was detected as evolving under neutral evolution with FUBAR, but without statistical significance.

\section{Molecular morphology reveals phylogenetic inertia of ICK peptides}

The 3D models of 41 calcin mature peptide sequences were generated using the 3D structure of imperacalcin, a calcin isolated from the venom of Pandinus imperator (Pdb file 1IE6) as a template. Additionally, to compare the morphology of calcins to that of the LKTx peptides, an additional 17 models were generated: 13 models for sequences from the family Buthidae, two from Chaerilidae, and two from Pseudochactidae. We characterized the accessible surface ratio (ASR) of the amino acid residues using GetArea (Fraczkiewicz \& Braun, 1998). Our results showed four of the six cysteines were buried forming the core of the protein (ASR less than 20\% exposed; Figs. S6-7), suggesting the disulfide bridges were not altered by the insertion/deletion of amino acids between cysteines (Fig. 2 b). Up to seven residues 
323 formed the core of the protein model of the LKTx peptides (Fig. S6). In contrast, five residues (four

324 cysteines) were buried forming the core of calcins (Fig. S7), although some calcins had up to seven

325 residues buried (the two species of Bothriurus).

326

327 To elucidate the evolution of these peptides, 3D models with solvent accessible surface area (SAS; Fig.

$3282 c$; Figs. S6-7) for two static image views (frontal and lateral) were generated using the APBS method

329 (Baker et al., 2001) and the PDB2PQR server (Dolinsky et al., 2004). Morphometric analysis of

330 molecular models showed that calcin shape is distinct from the LKTx peptides across multiple principal

331 components (Fig. 4). In the Elliptical Fourier Analysis (EFA) of the frontal SAS of calcins and LKTx

332 peptides, $49 \%$ of the variation is explained by PC1 $(p<0.01)$, and showed that calcins have a well-

333 defined apex (corresponding to extra amino acids before the first cysteine) and are consistently slenderer

334 than the LKTx, which are comparatively globular and lack the distinctive tip. PC2 explained 13\% of the

335 variation $(p=0.17)$ and showed the differences between the length of the left-anterior margin of the

336 calcins, and the right-anterior margin of the LKTx. For the lateral view, EFA of the lateral SAS of calcins

337 and LKTx, PC1 explained $67.5 \%$ of the variation $(p<0.01)$, and showed that calcins have two distinctive

338 apexes (top and bottom), whereas LKTx lack them. A comparatively globular shape is present on the

339 LKTx peptides in contrast with a slenderer shape of calcins. PC2 explained less than $10 \%$ of the variation

$340(p=0.42)$ and segregated the shape of three calcins based on the presence of a slender apex in their model

341 projection, as a result of a longer amino acid sequence (35, including more amino acids before the first

342 cysteine).

343

344 Superimposition of the phylogram onto the principal components of scorpion ICK peptides showed that

345 these homologs reflect the partitioning of molecular morphospace with high fidelity (Fig. $4 a, b$ ), and that

346 this partitioning mirrors the basal split between Buthida and Iurida. While calcins were segregated by PC1

347 of the frontal view of their 3D structure; LKTx peptides showed great shape plasticity across PC1, but

348 were more restricted in PC2. Furthermore, PC1 of the lateral view also distinguishes calcins from LKTx

349 peptides, but PC2 did not help separate these two groups. Phenograms of PC1 for both views also suggest

350 that the distinction between calcins and LKTx has persisted for a long span of evolutionary time (Fig. 4c,

$351 d)$.

352

353 Furthermore, the differences we observed in molecular shape between calcins and LKTx were not simply

354 a manifestation of the size of the molecule. We examined two other biochemical properties of peptides,

355 molecular weight and molecular volume. Phenograms of these properties showed broad overlap in the

356 molecular weight and volumes of calcins and LKTx peptides (Fig. S8-9). Similarly, we found no 
357 significant differences in the molecular weight $(t=-0.71 ; p=0.49)$ and molecular volume $(t=-0.84 ; p=$

358 0.41) of calcins and LKTx peptides, although we note that calcin molecular weight is less variable than

359 the molecular volume. These results suggest that the more globular shape of LKTx does not result from a

360 simple difference in the size of calcins and LKTx.

361

362 Further analyses support the inference that principal components of molecular shape are able to reflect

363 functional properties. We tested the correlation between PC1 of both surfaces and a third biochemical

364 property, net charge (Fig. 5). We discovered that net charge differs significantly between calcins and

$365 \operatorname{LKTx}(t=-12.04 ; p<0.01)$, and that the net charge is highly correlated with the molecular shape (Fig.

$3666 a, b$; Fig. S10). Heatmap comparison of the overall shape of calcins and LKTx identified the most

367 evolutionarily labile region of the peptide, noticeable both at sequence and morphological levels: the

368 amino acid residue ${ }^{29} \mathrm{R}$ (in our multiple sequence alignment, ${ }^{24} \mathrm{R}$ in calcin alignment only, Fig. $6 c, d$ ). This

369 amino acid residue forms the small lateral projection on the bottom of the molecule (absent in the LKTx

370 peptides). Using mutants of maurocalcin, Estève et al.(Estève et al., 2003) identified a critical role for this

371 arginine in binding the type 1 RyR (RyR1), because its replacement induced the complete loss of this

372 protein's effect on RyR.

373

374 To characterize evolutionary dynamics of ICK peptides, we also tested for significant shifts in

375 morphological regimes using the library $11 \mathrm{ou}+$ pBIC (Khabbazian et al., 2016). Eight shifts were

376 detected across the evolutionary history of ICK peptides, of which five were found in the LKTx peptides

377 of parvorder Buthida, and three in the calcins of the parvorder in Iurida (Fig. 7).

378

\section{Discussion}

380

381

Phylogenomic resolution of the scorpion tree of life

382

383

Maximum likelihood, species tree analyses, and Bayesian inference analyses supported the monophyly of the two basal clades in Scorpiones: Buthida and Iurida, a result recovered in a previous, more sparsely sampled phylogenomic analysis (Sharma et al., 2015). The internal relations within Buthidae were congruent with previous morphological hypothesis (Fet et al., 2003; Fet, Soleglad \& Lowe, 2005). By contrast, relationships within Iurida were congruent with more recent phylogenomic tree topologies, and maintain the non-monophyly of such groups as Chactoidea (Sharma et al., 2015, 2018). This molecular phylogeny included for the first time a member of the family Hemiscorpiidae, a lineage with a necrotoxic venom (unlike the neurotoxic venom characteristic of most Buthida). Its placement in the scorpion tree of 
391 life is nested deeply within a non-monophyletic Scorpionidae, contrary to previous hypotheses based on

392 morphology (Prendini, 2000). This suggests a recent evolutionary origin of necrotoxic venom in

393 scorpions (Fig. 1). The uniformity of these results suggested the trade-off between missing data, number

394 of genes, and species trees and supermatrix approaches does not have dramatic effects on the

395 reconstruction of the major clades within Scorpiones. The two libraries of Cercophonius squama were not

396 recovered as monophyletic. Given its current taxonomic status and broad distribution (New South Wales,

397 southwestern Australia, Queensland, and Tasmania), our results suggest this species could represent a

398 multispecies complex yet to be resolved.

399

400 Gene topology of ICK peptides reflects lineage-specific origins of calcins and LKTX

401

402 Our survey of ICK homologs greatly expanded their known representation across scorpion phylogeny

403 (Xiao et al., 2016; Santibáñez-López et al., 2016). The gene tree topology recovered herein showed

404 remarkable congruence with the phylogenomic tree (Figs. 1, 2a). Calcins were recovered as monophyletic

405 and phylogenetically restricted to Iurida. LKTx peptides were not recovered as monophyletic, but were

406 found to be phylogenetically restricted to Buthida (Buthidae, Chaerilidae, and Pseudochactidae).

407 Chaerilidae was previously thought to be a member of Iurida, and its placement with Buthidae and

408 Pseudochactidae was previously based only on molecular phylogenetic analyses. Thus, its transfer to

409 Buthida was not substantiated by any morphological characters. The gene tree topology recovered here

410 demonstrates the first known synapomorphy of Buthida as presently defined.

411

412 Selection regimes in ICK peptides support calcin and LKTx interact with conserved targets

413

414 Some authors (Sollod et al., 2005; Undheim et al., 2015) have suggested that the major problem in 415 reconstructing the molecular evolutionary histories of small peptides, particularly those cysteine-rich

416 peptides, is their conserved disulfide framework, because all non-cysteine amino acids can potentially

417 undergo substitutions without disturbing the protein core. Furthermore, it has been suggested that

418 disulfide bridges, by providing stability, should enable accelerated sequence evolution (by positive

419 selection) and act against deleterious mutations avoiding negative selection (Feyertag \& Alvarez-Ponce,

420 2017). Calcins and LKTx selection analyses showed that non-cysteine amino acids undergo few

421 substitutions in between cysteines, including insertion/deletions (Fig. 2b), and none evolving under

422 positive selection. Thus, our results support the hypothesis that non-CS $\alpha \beta$ toxins have evolved under the

423 influence of negative selection to preserve their coding sequences, suggesting they interact with

424 conserved targets (Sunagar et al., 2013). The highly conserved conformation of calcins, along with 
425 negative selection to reduce alternate states, is consistent with the tendency for small proteins to rely on

426 being folded into a stable conformation, i.e., to preserve their function (venom potency) rather than

427 structural integrity (Undheim, Mobli \& King, 2016). This is observed in the fractional conductance

428 induction to RyRs in eight calcins studied (Xiao et al., 2016). The conductance of these calcins ranged

429 from 0.35 to 0.60 , suggesting that their high structural similarity allows calcin to bind to RyRs at the same

430 site, engaging the receptive amino acid with varying degrees of affinity.

431

432 The high incidence of shifts in Buthida may partly reflect the asymmetrical diversity of this lineage, as the 433 family Buthidae alone comprises approximately half of all described scorpion species. Alternatively, it 434 may reflect some degree of evolutionary lability, as reflected by such metrics as LKTx molecular weight. 435 By comparison, all calcins share a conservative regime (in gray, Fig. 7) except for three non-converging 436 shifts restricted to recently diverging branches. Taken together, our analyses suggest that the evolutionary 437 dynamics of scorpion ICK homologs reflect the underlying phylogeny, not only at the level of sequence 438 data, but also in proxies of protein function.

439

440

\section{Calcin evolution within Iurida reflects phylogenetic signal, not classification}

441

442 According to our phylogenomic analyses, the present classification of Iurida includes numerous non443 monophyletic groups (e.g., Chactoidea, Chactidae, Scorpionidae, Hormuridae; Fig. 1). Intriguingly, EFA 444 analysis of calcins revealed a strong correspondence between the principal components of molecular 445 shape and the relationships recovered by our analyses. A vizualisation of morphospace within Iurida 446 paralleled the recovery of distinct clades recovered in the phylogenomic tree (Fig. S11). Within the 447 paraphyletic Chactoidea, we observed that each clade that renders the chactoids non-monophyletic 448 clustered in morphospace, frequently to the exclusion of other such clusters, for both the frontal and 449 lateral views. We also examined the morphospace for the relative clustering of Bothriuroidea and 450 Scorpionoidea, which were previously inferred to be part of a monophyletic superfamily (the traditionally 451 defined Scorpionoidea; Prendini, 2000)]. Consistent with phylogenomic results (Sharma et al., 2015, 452 2018), Bothriuroidea and Scorpionoidea occupied markedly different parts of morphospace (Fig. S11). 453

454 The backbone phylogeny of Iurida was previously not strongly supported and two different phylogenomic 455 datasets recovered different relationships within this group (Sharma et al., 2015; Starrett et al., 2017). 456 Both the paraphyly of Chactoidea and the subdivision of the erstwhile Scorpionoidea have been treated 457 with a reasonable degree of skepticism, given limitations in taxonomic sampling in phylogenomic studies 458 (Sharma et al., 2015; Starrett et al., 2017; Monod et al., 2017; Sharma et al., 2018), and thus the 
459

460

461

462

\section{Conclusion}

464

465

466

467

468

469

470

471

472

473

474

475

476

477

478

479

480

481

482

483

484

485

486

487

488

489

490

491

classification of scorpions likely retains many non-monophyletic taxa. Our results suggest that calcin sequence, as well as calcin shape, retain high phylogenetic signal in spite of selection (Fig. 2; Fig. S11), and accord with the molecular phylogeny, rather than scorpion classification.

The dataset we assembled here shows that a key single-copy ortholog of an ICK peptide was present in the common ancestor of scorpions. This ancestral peptide subsequently diversified into LKTx and calcins, in the two lineages originating from the basal split in scorpions. Taken together, our analyses suggest that the evolutionary dynamics of the ensuing scorpion ICK homologs reflect to a surprising degree the underlying phylogeny of this arachnid group, not only at the level of sequence data, but also in models of molecular shape and proxies of protein function (e.g., net charge). The evolutionary dynamics exhibited by calcins differ markedly from patterns described in various venomous animals. Comparable analyses of rattlesnake venom have shown that closely related species of Crotalus can possess different subsets of venom genes entirely, a dynamic partly driven by ancient radiation followed by gene loss (Fry et al., 2003; Dowell et al., 2016). Analyses of cone snail and spider venoms have also shown evidence for strong positive selection and extensive gene turnover, with high variance in copy number and elevated rates of nonsynonymous mutations (Binford et al., 2008; Garb \& Hayashi, 2013; Phuong, Mahardika \& Alfaro, 2016; Phuong \& Mahardika, 2018). Scorpion ICK homologs exhibit strikingly different phenomena, with little turnover within calcins or LKTxs, and a general pattern of negative selection. The evolutionary conservation of this gene family is all the more remarkable given the estimated Permian age of diversification of crown group scorpions (Sharma et al., 2018).

Our analysis of molecular shape provides the first synapomorphies for the well-supported, and recently redefined, clades Buthida (i.e., the presence of LKTx) and Iurida (i.e., the presence of calcins). These clades have heretofore proven difficult to define using anatomical characters; we were able to show that "molecular morphology" can overcome this limitation. To our knowledge, this work constitutes the first report of a synapomorphy defined from a molecule's shape in the study of arthropod phylogenetics.

\section{Acknowledgments}

We are greatly indebted to Ernesto Ortiz who kindly provided us with information on some of the webservers used here. Access to computing nodes provided by the Center for High Throughput Computing (CHTC) and the Bioinformatics Resource Center (BRC) of the University of Wisconsin- 
492 Madison. Comments from Kevin Arbuckle and two anonymous reviewers refined an earlier draft of the

493 manuscript.

494

495

496

497

498

499

500

501

502

503

504

505

506

507

508

509

510

511

512

513

514

515

516

517

518

519

520

521

522

523

524

525

\section{References}

Baker NA, Sept D, Joseph S, Holst MJ, McCammon JA 2001. Electrostatics of Nanosystems: Application to Microtubules and the Ribosome. Proceedings of the National Academy of Sciences of the United States of America 98:10037-10041.

Ballesteros JA, Hormiga G 2016. A New Orthology Assessment Method for Phylogenomic Data: Unrooted Phylogenetic Orthology. Molecular Biology and Evolution 33:2117-2134.

Biasini M, Bienert S, Waterhouse A, Arnold K, Studer G, Schmidt T, Kiefer F, Cassarino TG, Bertoni M, Bordoli L, Schwede T 2014. SWISS-MODEL: Modelling Protein Tertiary and Quaternary Structure Using Evolutionary Information. Nucleic acids research 42:W252-W258.

Binford GJ, Bodner MR, Cordes MHJ, Baldwin KL, Rynerson MR, Burns SN, Zobel-Thropp PA 2008. Molecular Evolution, Functional Variation, and Proposed Nomenclature of the Gene Family That Includes Sphingomyelinase D in Sicariid Spider Venoms. Molecular Biology and Evolution 26:547566.

Bonhomme V, Picq S, Gaucherel C, Claude J 2014. Momocs: Outline Analysis Using R. Journal of Statistical Software 56:1-24.

Capella-Gutiérrez S, Silla-Martínez JM, Gabaldón T 2009. trimAl: A Tool for Automated Alignment Trimming in Large-scale Phylogenetic Analyses. Bioinformatics 25:1972-1973.

Casewell NR, Wüster W, Vonk FJ, Harrison RA, Fry BG 2013. Complex Cocktails: the Evolutionary Novelty of Venoms. Trends in Ecology \& Evolution 28:219-229.

Coddington JA, Giribet G, Harvey MS, Prendini L, Walter DE 2004. Arachnida. In: Cracraft J, Donoghue PCJ eds. Assembling the Tree of Life, pp. 296-318. New York, NY: Oxford University Press.

Darriba D, Taboada GL, Doallo R, Posada D 2011. ProtTest 3: Fast Selection of Best-fit Models of Protein Evolution. Bioinformatics 27:1164-1165.

Dolinsky TJ, Nielsen JE, McCammon JA, Baker NA 2004. PDB2PQR: An Automated Pipeline for the Setup of Poisson-Boltzmann Electrostatics Calculations. Nucleic acids research 32:W665-W667. Dongen S 2000. Graph Clustering by low simulation. PhD Thesis. University of Utrecht.

Dowell NL, Giorgianni MW, Kassner VA, Selegue JE, Sanchez EE, Carroll SB 2016. The Deep Origin and Recent Loss of Venom Toxin Genes in Rattlesnakes. Current Biology 26:2434-2445.

Enright AJ, Van Dongen S, Ouzounis CA 2002. An Efficient Algorithm for Large-scale Detection of Protein Families. Nucleic acids research 30:1575-1584.

Estève E, Smida-Rezgui S, Sárközi S, Szegedi C, Regaya I, Chen L, Altafaj X, Rochat H, Allen P, Pessah 
526

527

528

529

530

531

532

533

534

535

536

537

538

539

540

541

542

543

544

545

546

547

548

549

550

551

552

553

554

555

556

557

558

559

IN, Marty I, Sabatier J-M, Jóna I, De Waard M, Ronjat M 2003. Critical Amino Acid Residues

Determine the Binding Affinity and the $\mathrm{Ca}^{2+}$ Release Efficacy of Maurocalcine in Skeletal Muscle Cells. Journal of Biological Chemistry 278:37822-37831.

Fet V, Gantenbein B, Gromov A, Lowe G, Lourenço WR 2003. The First Molecular Phylogeny of Buthidae (Scorpiones). Euscorpius 4:1-10.

Fet V, Soleglad ME, Lowe G 2005. A New Trichobotrhial Character for the High-level Systematics of Buthoidea (Scorpiones: Buthida). Euscorpius 23:1-40.

Feyertag F, Alvarez-Ponce D 2017. Disulfide Bonds Enable Accelerated Protein Evolution. Molecular Biology and Evolution 34:1833-1837.

Fraczkiewicz R, Braun W 1998. Exact and Efficient Analytical Calculation of the Accessible Surface Areas and Their Gradients for Macromolecules. Journal of Computational Chemistry 19:319-333.

Fry BG, Roelants K, Champagne DE, Scheib H, Tyndall JDA, King GF, Nevalainen TJ, Norman JA, Lewis RJ, Norton RS, Renjifo C, la Vega de RCR 2009. The Toxicogenomic Multiverse: Convergent Recruitment of Proteins Into Animal Venoms. Annual Review of Genomics and Human Genetics $10: 483-511$.

Fry BG, W ster W, Kini RM, Brusic V, Khan A, Venkataraman D, Rooney AP 2003. Molecular Evolution and Phylogeny of Elapid Snake Venom Three-Finger Toxins. Journal of molecular evolution 57:110-129.

Gao B, Harvey PJ, Craik DJ, Ronjat M, De Waard M, Zhu S 2013. Functional Evolution of Scorpion Venom Peptides with an Inhibitor Cystine Knot Fold. Bioscience Reports 33:513-527.

Garb JE, Hayashi CY 2013. Molecular Evolution of $\alpha$-Latrotoxin, the Exceptionally Potent Vertebrate Neurotoxin in Black Widow Spider Venom. Molecular Biology and Evolution 30:999-1014.

Grabherr MG, Haas BJ, Yassour M, Levin JZ, Thompson DA, Amit I, Adiconis X, Fan L, Raychowdhury R, Zeng Q, Chen Z, Mauceli E, Hacohen N, Gnirke A, Rhind N, di Palma F, Birren BW, Nusbaum C, Lindblad-Toh K, Friedman N, Regev A 2011. Full length transcriptome assembly from RNA-Seq data without a reference genome. Nature Biotechnology 29:644-652.

Haney RA, Clarke TH, Gadgil R, Fitzpatrick R, Hayashi CY, Ayoub NA, Garb JE 2016. Effects of Gene Duplication, Positive Selection, and Shifts in Gene Expression on the Evolution of the Venom Gland Transcriptome in Widow Spiders. Genome Biology and Evolution 8:228-242.

Herzig V, Wood DLA, Newell F, Chaumeil PA, Kaas Q, Binford GJ, Nicholson GM, Gorse D, King GF 2010. ArachnoServer 2.0, an Updated Online Resource for Spider Toxin Sequences and Structures. Nucleic acids research 39:D653-D657.

Jeram AJ 2005. Phylogeny, Classification and Evolution of Silurian and Devonian Scorpions. In: Selden, PA, ed. Proceedings of the $17^{\text {th }}$ European Colloquium of Arachnology, pp. 1-15. Edinburgh, UK. 
560 Juarez P, Comas I, Gonzalez-Candelas F, Calvete JJ 2008. Evolution of Snake Venom Disintegrins by

561 Positive Darwinian Selection. Molecular Biology and Evolution 25:2391-2407.

562 Katoh K, Standley DM 2013. MAFFT Multiple Sequence Alignment Software Version 7: Improvements

563 in Performance and Usability. Molecular Biology and Evolution 30:772-780.

564 Khabbazian M, Kriebel R, Rohe K, Ané C 2016. Fast and Accurate Detection of Evolutionary Shifts in

565 Ornstein-Uhlenbeck Models. Methods in Ecology and Evolution 7:811-824.

566 King GF, Hardy MC 2013. Spider-Venom Peptides: Structure, Pharmacology, and Potential for Control

567 of Insect Pests. Annual review of entomology 58:475-496.

568 Kjellesvig-Waering EN 1986. A restudy of the Fossil Scorpionida of the World. Palaeontographica 569 Americana 55:1-287.

570 Kozlov AM, Aberer AJ, Stamatakis A 2015. ExaML version 3: A Tool for Phylogenomic Analyses on 571 Supercomputers. Bioinformatics 31:2577-2579.

572 Lartillot N, Rodrigue N, Stubbs D, Richer J 2013. PhyloBayes MPI: Phylogenetic Reconstruction with

573 Infinite Mixtures of Profiles in a Parallel Environment. Systematic Biology 62:611-615.

574 Lee CW, Lee EH, Takeuchi K, Takahashi H, Shimada I, Sato K, Shin SY, Kim DH, Kim JI 2004.

575 Molecular Basis of the High-affinity Activation of Type 1 Ryanodine Receptors by Imperatoxin A.

576 Biochemical Journal 377:385-394.

577 Mabrouk K, Ram N, Boisseau S, Strappazzon F, Rehaim A, Sadoul R, Darbon H, Ronjat M, De Waard M

578 2007. Critical Amino Acid Residues of Maurocalcine Involved in Pharmacology, Lipid Interaction

579 and Cell Penetration. Biochimica et Biophysica Acta (BBA) - Biomembranes 1768:2528-2540.

580 Meusemann K, Reumont von BM, Simon S, Roeding F, Strauss S, Kück P, Ebersberger I, Walzl M, Pass

581 G, Breuers S, Achter V, Haeseler von A, Burmester T, Hadrys H, Wägele JW, Misof B 2010. A

582 Phylogenomic Approach to Resolve the Arthropod Tree of Life. Molecular Biology and Evolution $583 \quad 27: 2451-2464$.

584 Minh BQ, Nguyen MAT, Haeseler von A 2013. Ultrafast Approximation for Phylogenetic Bootstrap.

$585 \quad$ Molecular Biology and Evolution 30:1188-1195.

586 Mirarab S, Warnow T 2015. ASTRAL-II: Coalescent-based Species Tree Estimation with Many

587 Hundreds of Taxa and Thousands of Genes. Bioinformatics 31:44-52.

588 Monod L, Cauwet L, González-Santillán E, Huber S 2017. The Male Sexual Apparatus in the Order

589 Scorpiones (Arachnida): A Comparative Study of Functional Morphology as a Tool to Define

$590 \quad$ Hypotheses of Homology. Frontiers in Zoology 14:1-48.

591 Murrell B, Moola S, Mabona A, Weighill T, Sheward D, Kosakovsky Pond SL, Scheffler K 2013.

592 FUBAR: A Fast, Unconstrained Bayesian AppRoximation for Inferring Selection. Molecular Biology 593 and Evolution 30:1196-1205. 
594 Murrell B, Wertheim JO, Moola S, Weighill T, Scheffler K, Kosakovsky Pond SL 2012. Detecting

595 Individual Sites Subject to Episodic Diversifying Selection. PLoS Genetics 8:e1002764.

596 Nguyen L-T, Schmidt HA, Haeseler von A, Minh BQ 2014. IQ-TREE: A Fast and Effective Stochastic

597 Algorithm for Estimating Maximum-Likelihood Phylogenies. Molecular Biology and Evolution

$598 \quad 32: 268-274$.

599 Orengo CA, Jones DT, Thornton JM 1994. Protein Superfamilies and Domain Superfolds. Nature $600 \quad 372: 631-634$.

601 Paradis E 2013. Molecular Dating of Phylogenies by Likelihood Methods: A Comparison of Models and 602 a New Information Criterion. Molecular Phylogenetics and Evolution 67:436-444.

603 Paradis E, Claude J, Strimmer K 2004. APE: Analyses of Phylogenetics and Evolution in R language. 604 Bioinformatics 20:289-290.

605 Phuong MA, Mahardika GN 2018. Targeted Sequencing of Venom Genes from Cone Snail Genomes 606 Improves Understanding of Conotoxin Molecular Evolution. Molecular Biology and Evolution $607 \quad 35: 1210-1224$.

608 Phuong MA, Mahardika GN, Alfaro ME 2016. Dietary Breadth is Positively Correlated with Venom 609 Complexity in Cone Snails. BMC Genomics:1-15. DOI: 10.1186/s12864-016-2755-6.

610 Possani LD, Becerril B, Delepierre M, Tytgat J 1999. Scorpion Toxins Specific for Nat-channels. The 611 FEBS Journal 264:287-300.

612 Prendini L 2000. Phylogeny and Classification of the Superfamily Scorpionoidea Latreille 1802 613 (Chelicerata, Scorpiones): An Exemplar Approach. Cladistics 16:1-78.

614 Price MN, Dehal PS, Arkin AP 2010. FastTree 2--approximately Maximum-likelihood Trees for Large 615 Alignments. PLoS ONE 5:e9490.

616 Ram N, Aroui S, Jaumain E, Bichraoui H, Mabrouk K, Ronjat M, Lortat-Jacob H, De Waard M 2008.

617 Direct Peptide Interaction with Surface Glycosaminoglycans Contributes to the Cell Penetration of 618 Maurocalcine. Journal of Biological Chemistry 283:24274-24284.

619 Revell LJ, 2012. phytools: an R package for phylogenetic comparative biology (and other things).

620 Methods in Ecology and Evolution 3:217-223.

621 Rokyta DR, Wray KP, Lemmon AR, Lemmon EM, Caudle SB 2011. A High-throughput Venom-gland 622 Transcriptome for the Eastern Diamondback Rattlesnake (Crotalus adamanteus) and Evidence for 623 Pervasive Positive Selection Across Toxin Classes. Toxicon 57:657-671.

624 Ronjat M, Feng W, Dardevet L, Dong Y, Khoury Al S, Chatelain FC, Vialla V, Chahboun S, Lesage F, 625 Darbon H, Pessah IN, De Waard M 2016. In Cellulo Phosphorylation Induces Pharmacological 626 Reprogramming of Maurocalcin, a Cell-penetrating Venom Peptide. Proceedings of the National 627 Academy of Sciences of the United States of America 113:E2460-8. 
628 Ronquist F, Teslenko M, van der Mark P, Ayres DL, Darling A, Höhna S, Larget B, Liu L, Suchard MA, 629 Huelsenbeck JP 2012. MrBayes 3.2: Efficient Bayesian Phylogenetic Inference and Model Choice 630 Across a Large Model Space. Systematic Biology 61:539-542.

631 Sanderson MJ 2002. Estimating Absolute Rates of Molecular Evolution and Divergence times: A

632 Penalized Likelihood Approach. Molecular Biology and Evolution 19:101-109.

633 Santibáñez-López CE, Possani LD 2015. Overview of the Knottin Scorpion Toxin-like Peptides in 634 Scorpion Venoms: Insights on Their Classification and Evolution. Toxicon 107:317-326.

635 Santibáñez-López C, Cid-Uribe J, Batista C, Ortiz E, Possani L 2016. Venom Gland Transcriptomic and 636 Proteomic Analyses of the Enigmatic Scorpion Superstitionia donensis (Scorpiones:

637 Superstitioniidae), with Insights on the Evolution of Its Venom Components. Toxins 8:367-24.

638 Santibáñez-López CE, Cid-Uribe JI, Zamudio FZ, Batista CVF, Ortiz E, Possani LD 2017. Venom Gland 639 Transcriptomic and Venom Proteomic Analyses of the Scorpion Megacormus gertschi Díaz-Najera, 6401966 (Scorpiones: Euscorpiidae: Megacorminae). Toxicon 133:95-109.

641 Santibanez Lopez CE, Kriebel R, Sharma PP 2017. eadem figura manet: Measuring Morphological 642 Convergence in Diplocentrid Scorpions (Arachnida: Scorpiones: Diplocentridae) under a Multilocus 643 Phylogenetic Framework. Invertebrate Systematics 31:233-16.

644 Sharma PP, Kaluziak ST, Pérez-Porro AR, González VL, Hormiga G, Wheeler WC, Giribet G 2014. 645 Phylogenomic Interrogation of Arachnida Reveals Systemic Conflicts in Phylogenetic Signal. $646 \quad$ Molecular Biology and Evolution 31:2963-2984.

647 Sharma PP, Fernández R, Esposito LA, González-Santillán E, Monod L 2015. Phylogenomic Resolution 648 of Scorpions Reveals Multilevel Discordance with Morphological Phylogenetic Signal. Proceedings 649 of the Royal Society Biological sciences 282:20142953.

650 Sharma PP, Baker CM, Cosgrove JG, Johnson JE, Oberski JT, Raven RJ, Harvey MS, Boyer SL, Giribet 651 652 653 654 655 656 G 2018. A Revised Dated Phylogeny of Scorpions: Phylogenomic Support for Ancient Divergence of 52 the Temperate Gondwanan Family Bothriuridae. Molecular Phylogenetics and Evolution 122:1-42.

Smith JJ, Vetter I, Lewis RJ, Peigneur S, Tytgat J, Lam A, Gallant EM, Beard NA, Alewood PF, Dulhunty AF 2013. Multiple Actions of f-LITX-Lw1a on Ryanodine Receptors Reveal a Functional Link Between Scorpion DDH and ICK toxins. Proceedings of the National Academy of Sciences of the United States of America 110:8906-8911.

Smith MD, Wertheim JO, Weaver S, Murrell B, Scheffler K, Kosakovsky Pond SL 2015. Less is More: an Adaptive Branch-site Random Effects Model for Efficient Detection of Episodic Diversifying 660 Selection. Molecular Biology and Evolution 32:1342-1353.

Soleglad ME, Fet V 2003. High-level Systematics and Phylogeny of the Extant Scorpions (Scorpiones:

$$
\text { Orthosterni). Euscorpius 11:1-210. }
$$


662 Sollod BL, Wilson D, Zhaxybayeva O, Gogarten JP, Drinkwater R, King GF 2005. Were Arachnids the 663 First to Use Combinatorial Peptide Libraries? Peptides 26:131-139.

664 Starrett J, Derkarabetian S, Hedin M, Bryson RW Jr., McCormack JE, Faircloth BC 2017. High

665 Phylogenetic Utility of an Ultraconserved Element Probe Set Designed for Arachnida. Molecular 666 Ecology Resources 17:812-823.

667 Sunagar K, Undheim E, Chan A, Koludarov I, Muñoz-Gómez S, Antunes A, Fry B 2013. Evolution 668 Stings: The Origin and Diversification of Scorpion Toxin Peptide Scaffolds. Toxins 5:2456-2487.

669 Suyama M, Torrents D, Bork P 2006. PAL2NAL: Robust Conversion of Protein Sequence Alignments $670 \quad$ into the Corresponding Codon Alignments. Nucleic acids research 34:W609-W612.

671 Tange O 2011. GNU Parallel - The Command-Line Power Tool. ;login The USENIX Magazine.

672 Undheim EAB, Grimm LL, Low C-F, Morgenstern D, Herzig V, Zobel-Thropp P, Pineda SS, Habib R, 673 Dziemborowicz S, Fry BG, Nicholson GM, Binford GJ, Mobli M, King GF 2015. Weaponization of 674 a Hormone: Convergent Recruitment of Hyperglycemic Hormone into the Venom of Arthropod 675 Predators. STFODE 23:1283-1292.

676 Undheim EAB, Mobli M, King GF 2016. Toxin Structures as Evolutionary Tools: Using Conserved 3D

677 Folds to Study the Evolution of Rapidly Evolving Peptides. BioEssays 38:539-548.

678 Waddington J, Rudkin DM, Dunlop JA 2015. A New mid-Silurian Aquatic Scorpion: One Step Closer to 679 Land? Biology Letters 11:20140815.

680 Weaver S, Shank SD, Spielman SJ, Li M, Muse SV, Kosakovsky Pond SL 2018. Datamonkey 2.0: A

681 Modern Web Application for Characterizing Selective and Other Evolutionary Processes. Molecular 682 Biology and Evolution 35:773-777.

683 Willard L, Ranjan A, Zhang H, Monzavi H, Boyko RF, Sykes BD, Wishart DS 2003. VADAR: A Web 684 Server for Quantitative Evaluation of Protein Structure Quality. Nucleic acids research 31:33166853319.

686 Wong ESW, Belov K 2012. Venom Evolution Through Gene Duplications. Gene 496:1-7.

687 Xiao L, Gurrola GB, Zhang J, Valdivia CR, SanMartin M, Zamudio FZ, Zhang L, Possani LD, Valdivia 688 HH 2016. Structure-Function Relationships of Peptides Forming the Calcin Family of Ryanodine 689 Receptor Ligands. The Journal of General Physiology 147:375-394. 


\section{Figure 1}

Scorpion tree of life

(a) Maximum likelihood tree topology recovered from the analysis of 3110 orthogroups, with 56 scorpion species and 13 outgroups. Bars to the right of terminals indicate number of orthologs. Shaded squares in Navajo plots indicate recovery of the node in the corresponding analysis with $M=$ Matrix followed by its number (except Mle=Matrix 1 analyzed with ExaML), and colored as follows: blue squares=IQTree; pink square=ASTRAL (see also Supplemental fig. 1). Representative scorpion species from the two parvorders: (b) adult male Troglokhammouanus steineri; (c) adult female Isometrus sp.; (d) adult male lurus dekanum; (e) adult male Superstitionia donensis; (f) subadult female Liocheles australasiae; (g) adult male Opisthacanthus madagascariensis; (h) adult female Kolotl magnus; and (i) adult female Megacormus gertschi. Photographs (b-f) by Gonzalo Giribet (with permission), and ( $g-h$ ) by Carlos E. Santibáñez-López. 
(a)

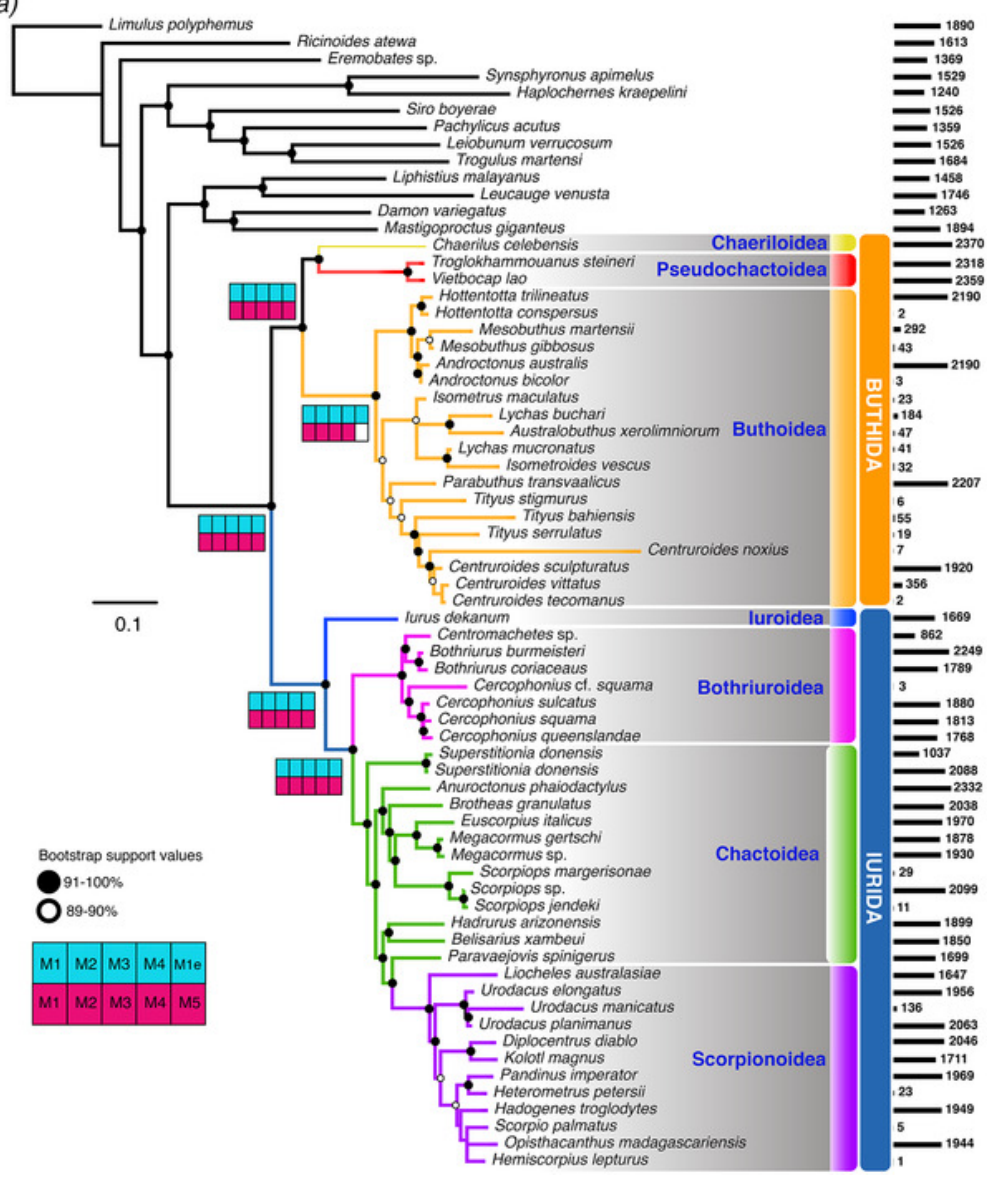

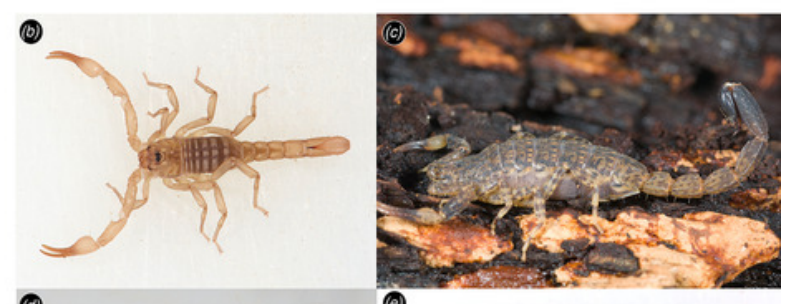

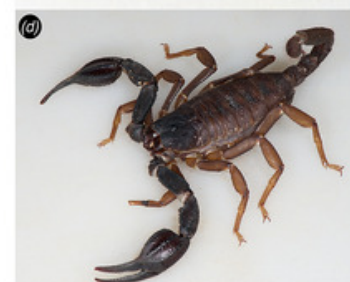

(ㄷ)
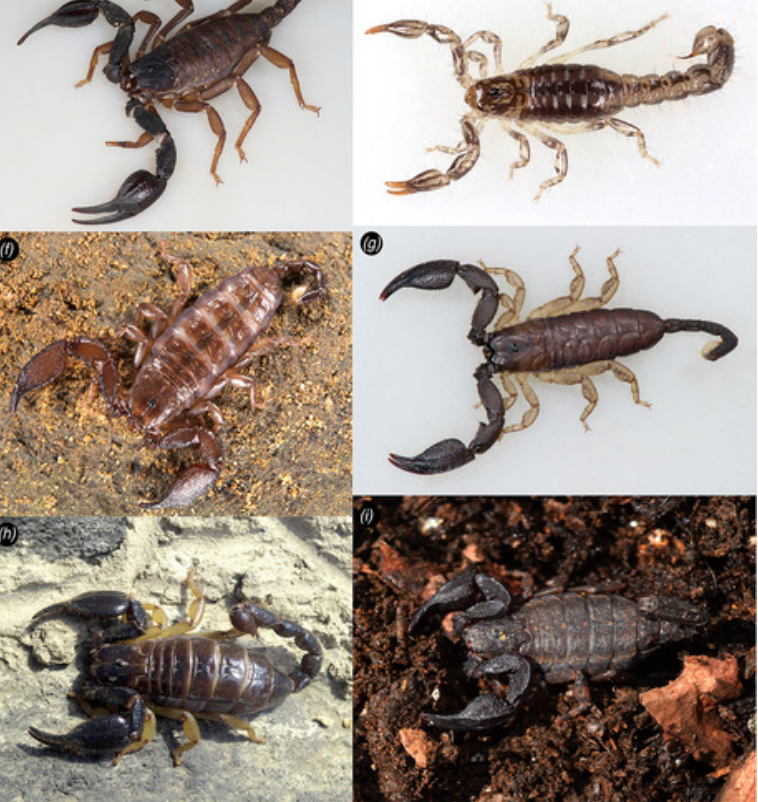


\section{Figure 2}

Calcin and LKTx evolutionary analyses

(a) Evolutionary tree topology of the ICK peptides recovered from the Bayesian inference analysis of 59 sequences of LKTx (Buthida) and calcins (lurida) isolated or deduced from cDNA cloned from venom of scorpion species. Consensus amino acid sequences of LKTX (b-d) and calcins (e) showing the highly-conserved disulfide bridges (lines) formed by six cysteines. Cysteines in red have their ASR less than 20\% exposed, cysteines in orange have their ASR more than $30 \%$ but less than 50\% exposed, and cysteines in blue have their ASR more than $51 \%$ exposed. Representative three-dimensional model of a calcin projected with the SAS corrected with APBS; frontal $(f)$ and lateral $(g)$ surfaces. Key amino acid used as landmarks to align the images indicated by their single letter code and their position in the MSA. 
(a)

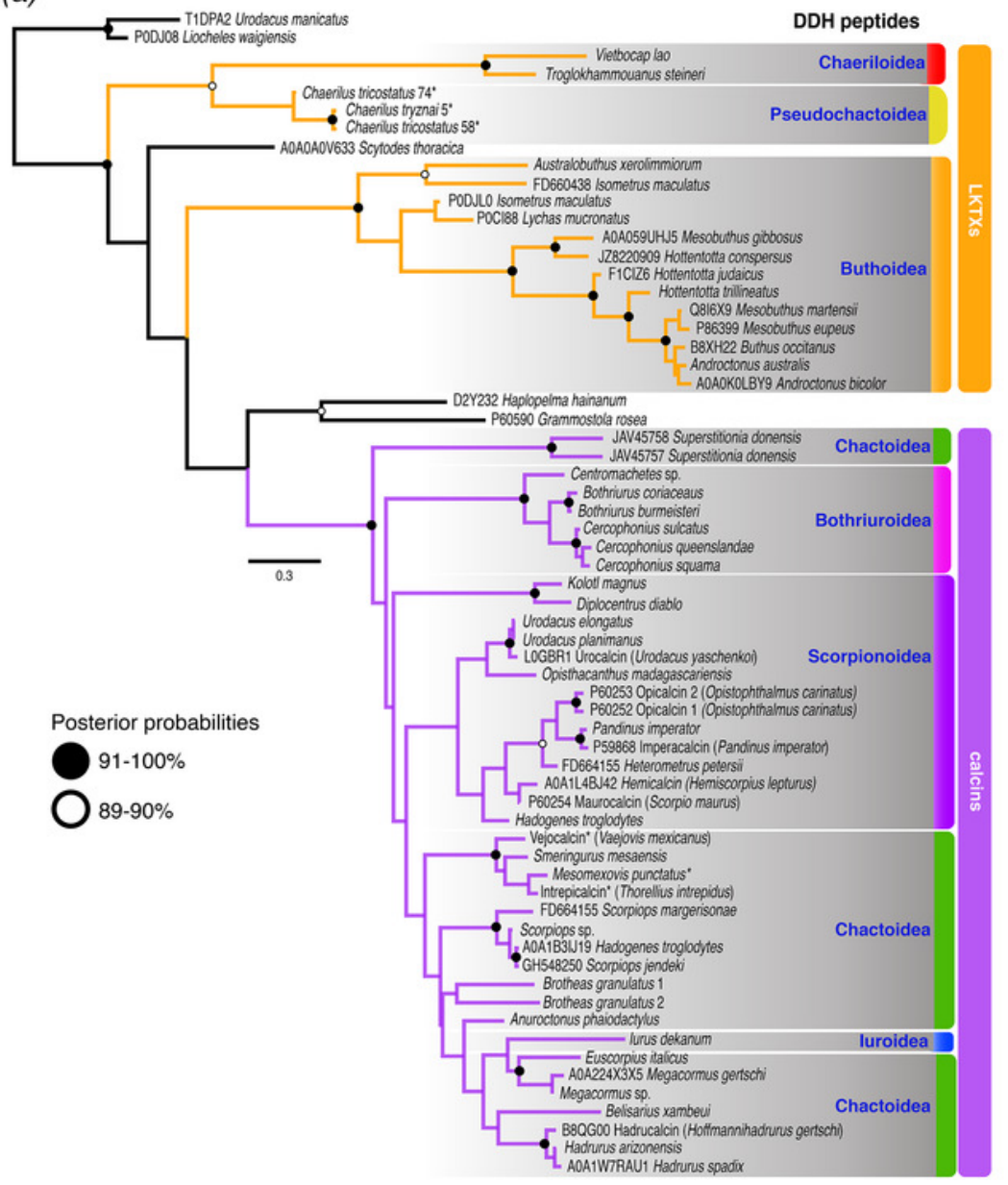

(b)

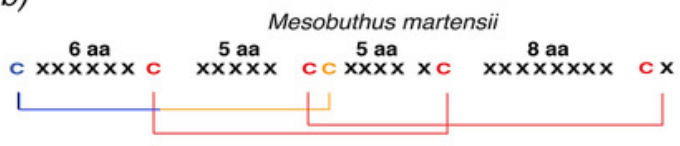

(c) Chaerilus tricostatus

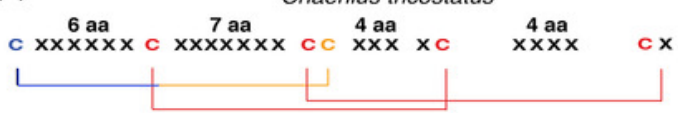

(d) Troglokhammouanus steineri

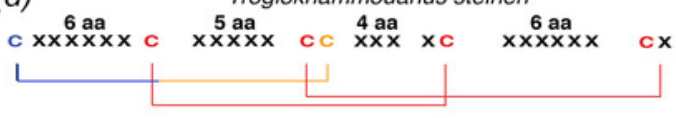

(e) Pandinus imperator

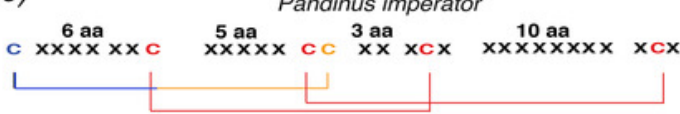

(f)

(g)

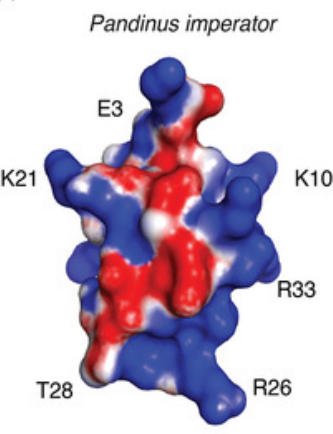

Pandinus imperator

R13

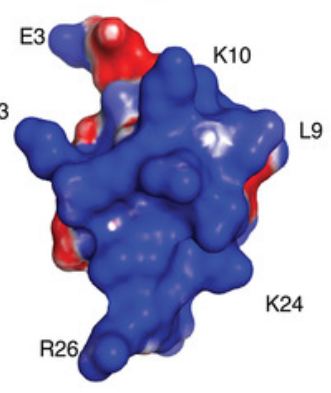




\section{Figure 3}

Molecular evolutionary analyses

$(a, b)$ Site selection analyses of the LKTx (a) and calcin $(b)$ sequences with FUBAR.

Visualization of the difference between the values of $\alpha$ (red) and $\beta$ (cyan). Light red indicates sites evolving under negative selection with probabilities greater than 0.90. $(c, d)$ Site selection analyses of the LKTX $(c)$ and calcin $(d)$ sequences with MEME as a function of the visualization of the difference between the values of $\alpha$ (synonymous substitution ratio, in red) and $b+$ (non-synonymous substitution at a site for the positive/neutral evolution component in cyan). Asterisk indicates the site with $\beta+$ value greater than $\alpha$ with a $p>0.90$. Other statistics are found on Tables S3-4. 
(a)

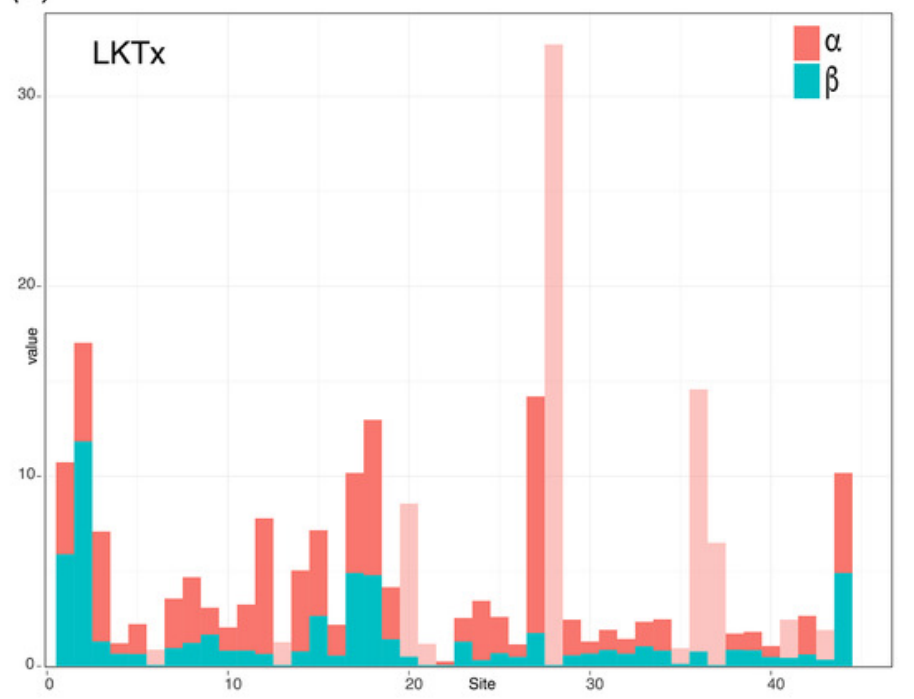

(c)

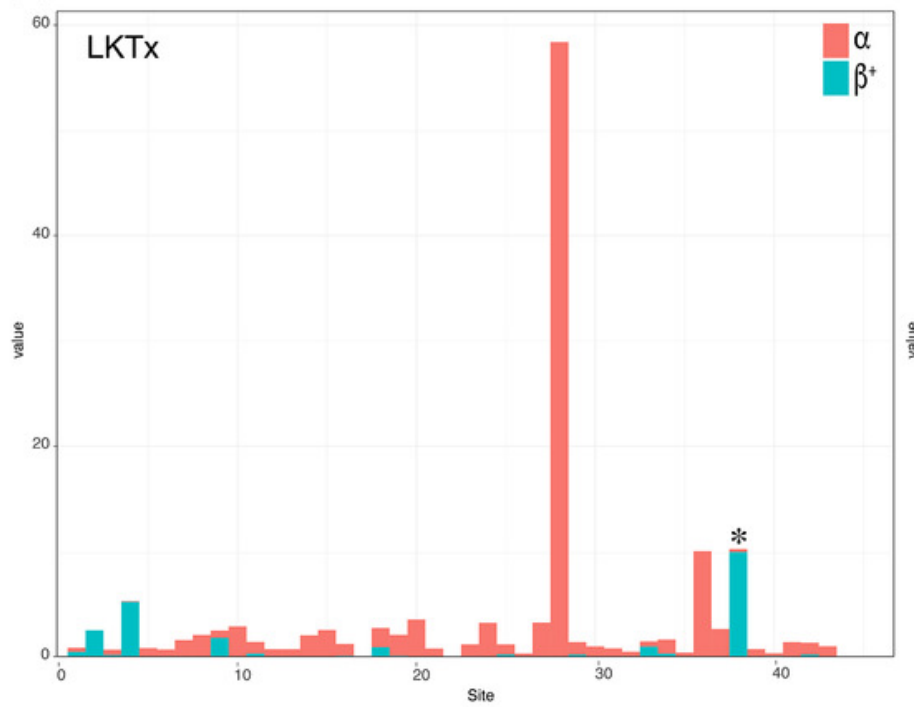

(b)

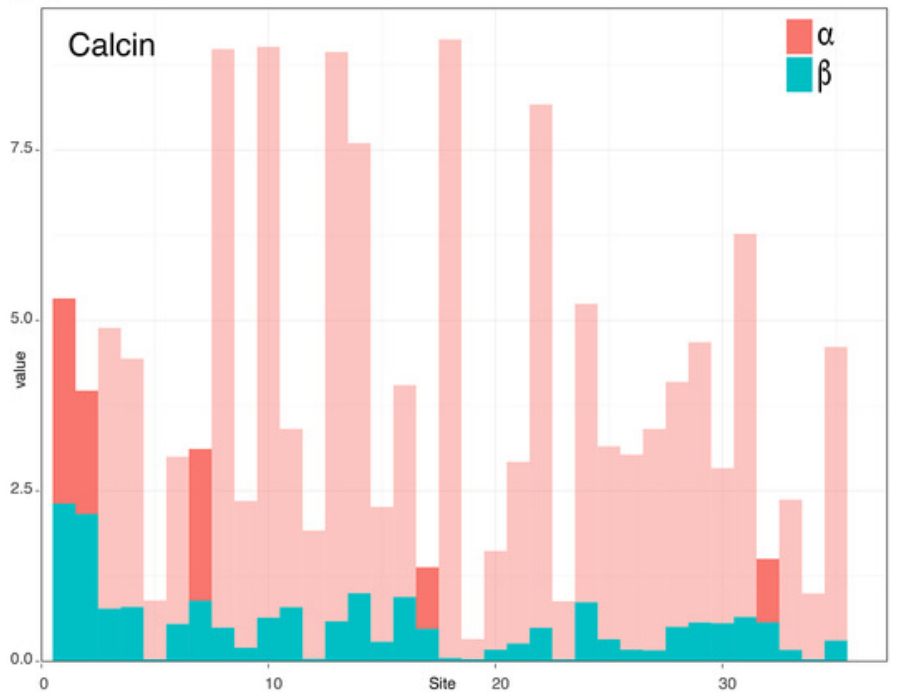

(d)

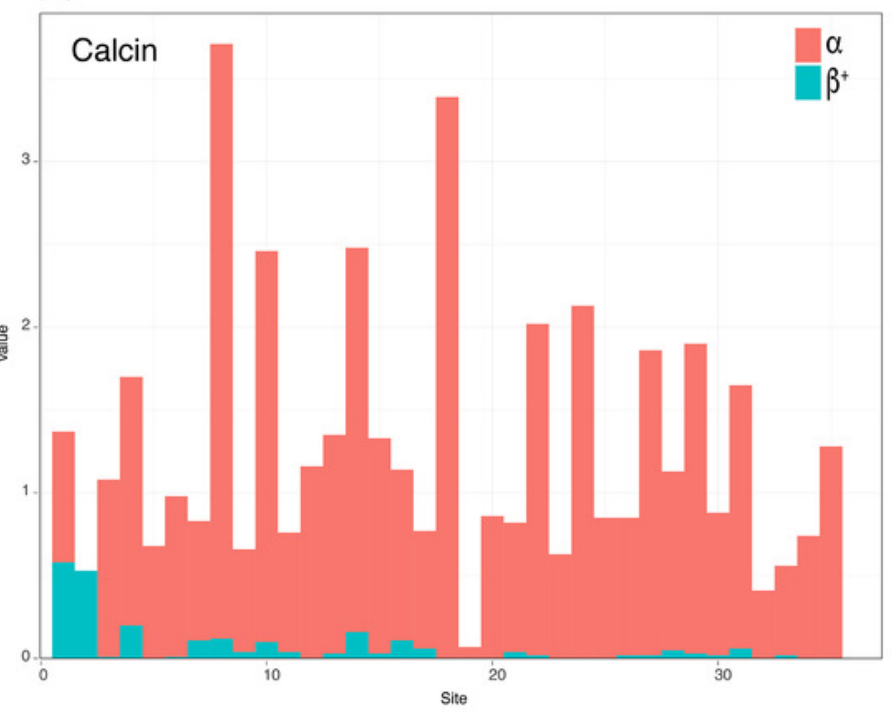




\section{Figure 4}

Morphometric analyses of the 3D structure of ICK peptides in scorpion venom

$(a, b)$ Visualisation of phylogenomic tree on the morphospace of the frontal $(a)$ and lateral $(b)$ shape data, showing the distinction between LTKx peptides (orange) and calcins (purple); horizontal axis indicates PC1 values and vertical axis indicates PC2 values. $(c, d)$ Visualization of PC1 values of the frontal $(c)$ and lateral $(d)$ shape data as a function of phylogenetic relationships recovered from the dated molecular tree; horizontal axis indicates the time of divergence and vertical axe indicates the $\mathrm{PCl}$ values.

(a)
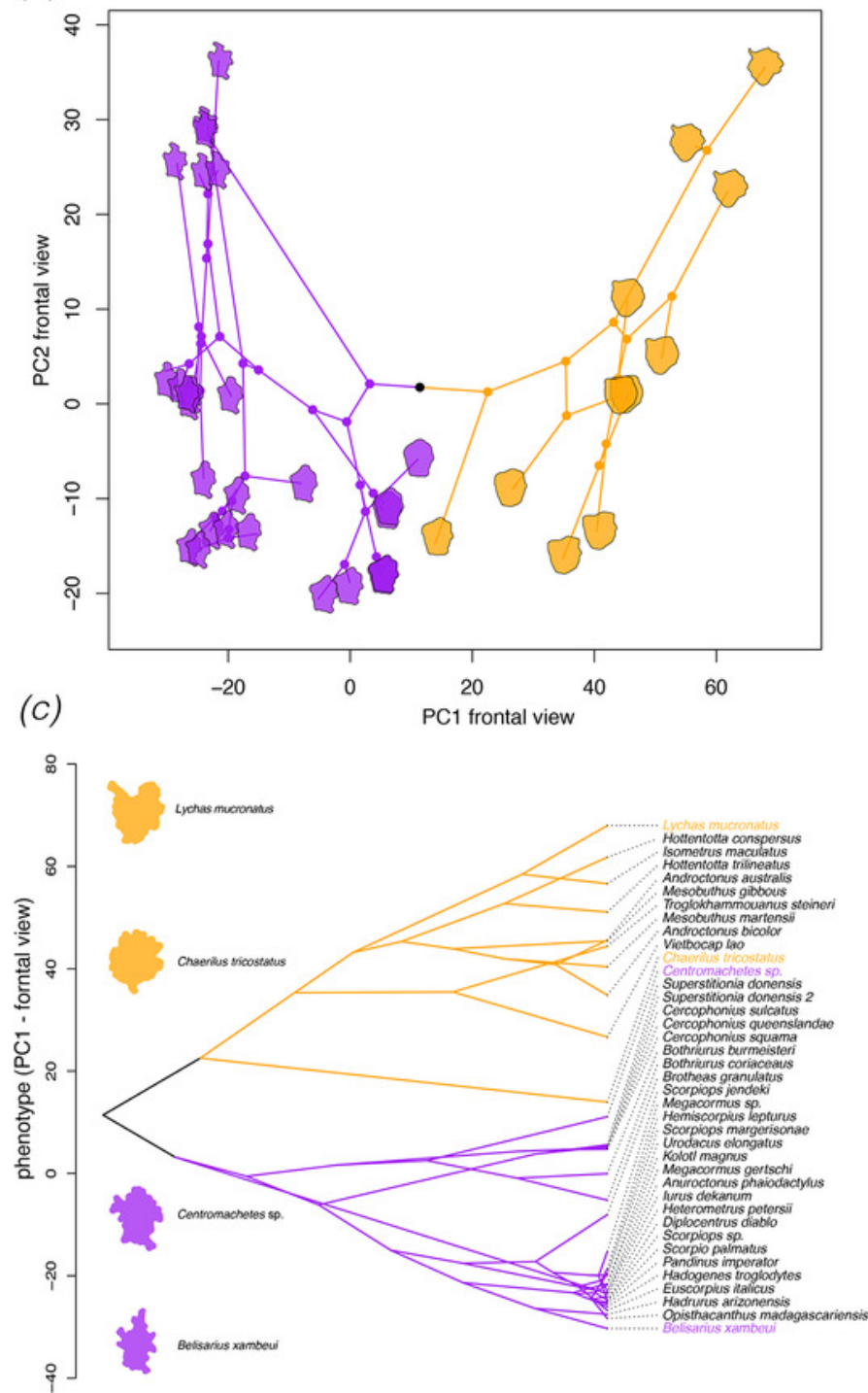

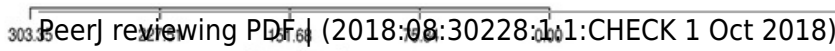
time (mya) (b)
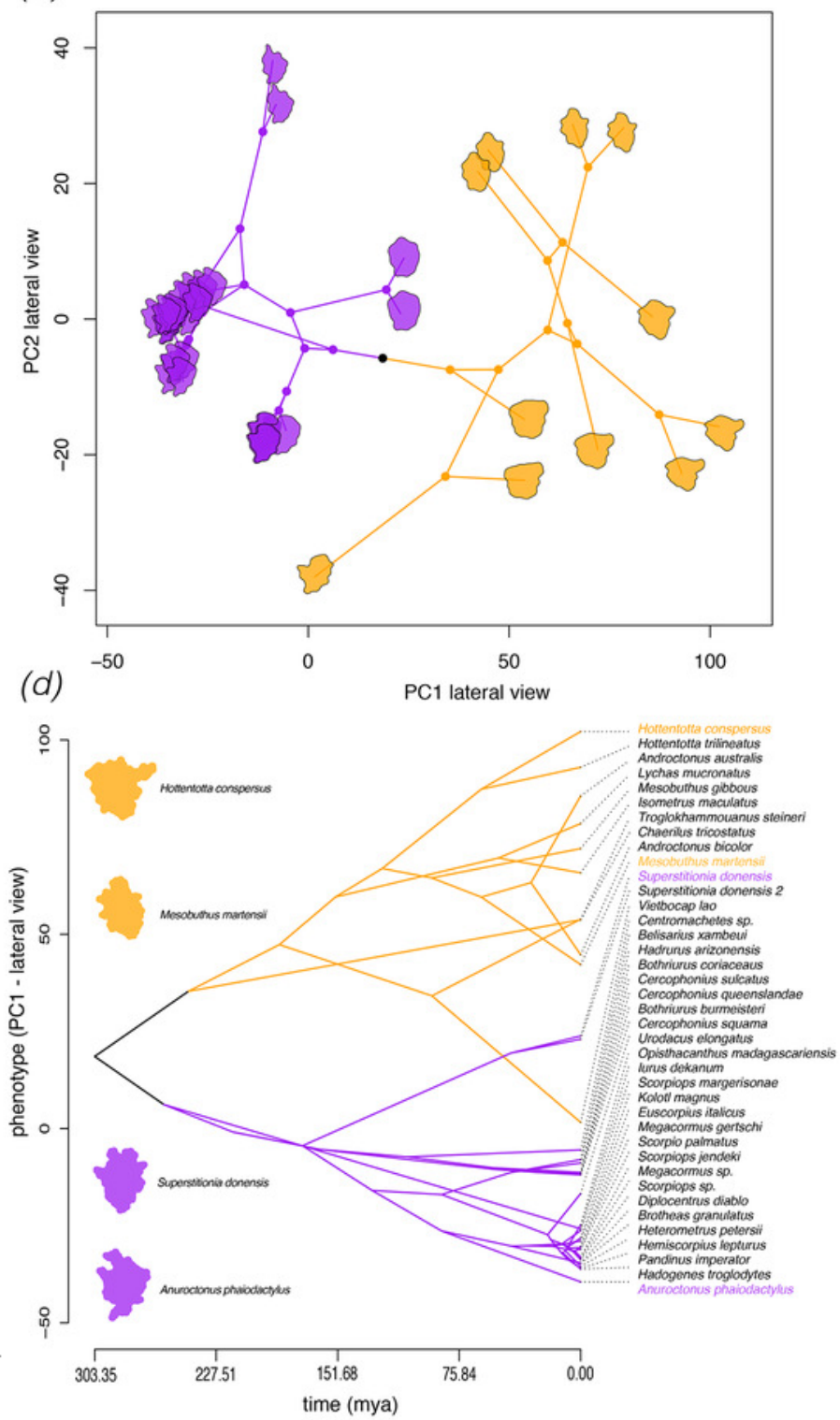


\section{Figure 5}

Correlation test between molecular shape and chemical properties

Correlation tests between molecular shape and chemical properties (a) Kendall rank correlation between molecular shape (PC both sides), weight, volume and net charge, color of the circle indicates positive or negative correlation coefficient, increasing size of the circle indicates smaller $p$-value. Kendall rank correlation between Net charge and (b) PC1 front, or (c) PCl side. r: correlation coefficient.

(a)

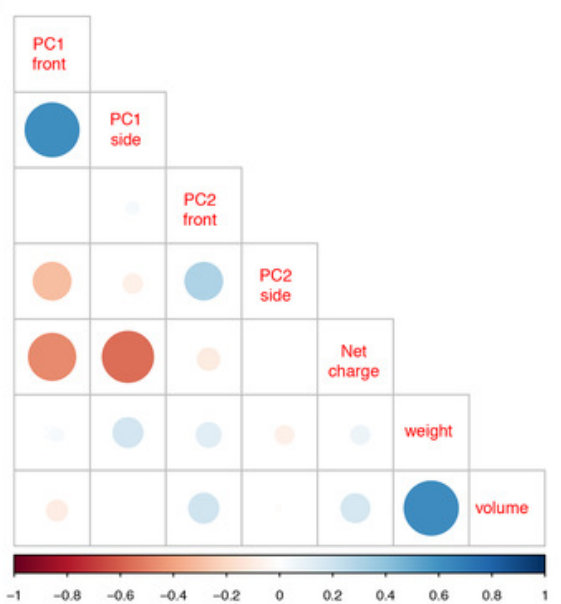

(b)

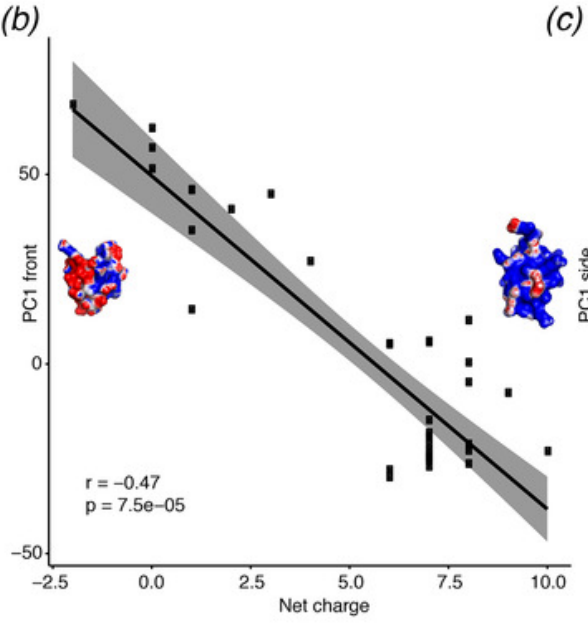

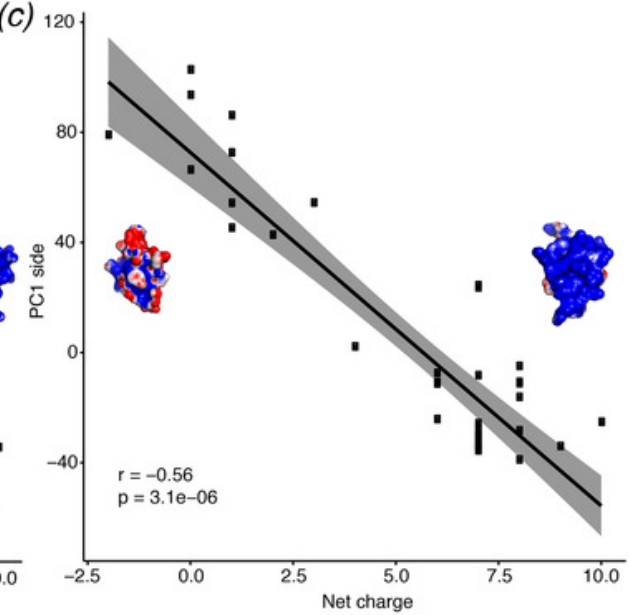




\section{Figure 6}

Evolutionary analyses on the molecular shape and the net charge

(a) Visualization of net charge values of calcins (purple) and LKTX (orange) as a function of phylogenetic distance (recovered from the dated molecular tree); horizontal axis indicates the time of divergence and vertical axis indicates the net charge values. (b) Phylomorphospace in three dimensions of PC1 values of the frontal and lateral views, and net charge. (c) Heatmap of the frontal (left) and lateral (right) views of calcin and LKTx peptides with major morphological differences represented by warm colors. Outlines represent the mean shape; numbers indicate the amino acid position in the multiple sequence alignment (MSA). (d) MSA of LTKx and calcin peptides, highlighting in squares the amino acid residues responsible for the major morphological differences shown as warm areas on heatmaps (c).

(a)

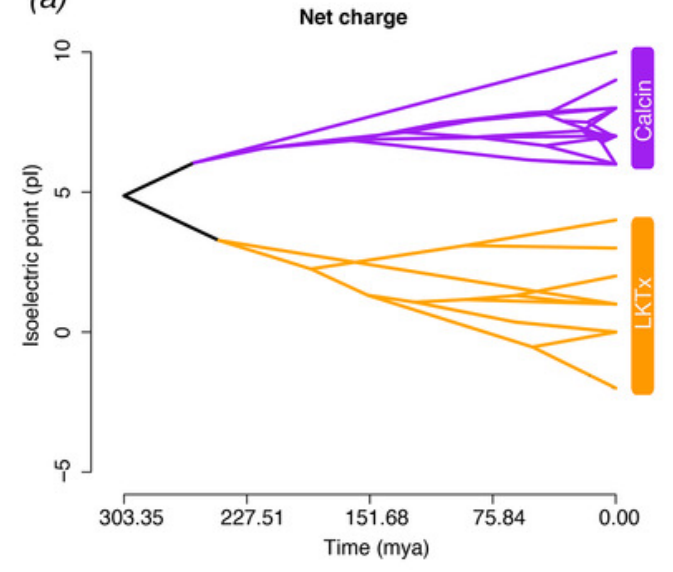

(c)

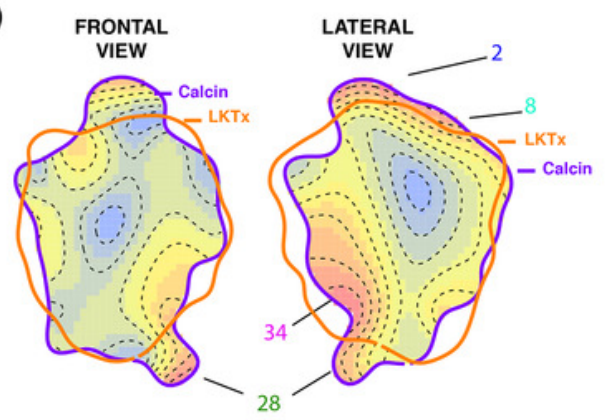

(b)

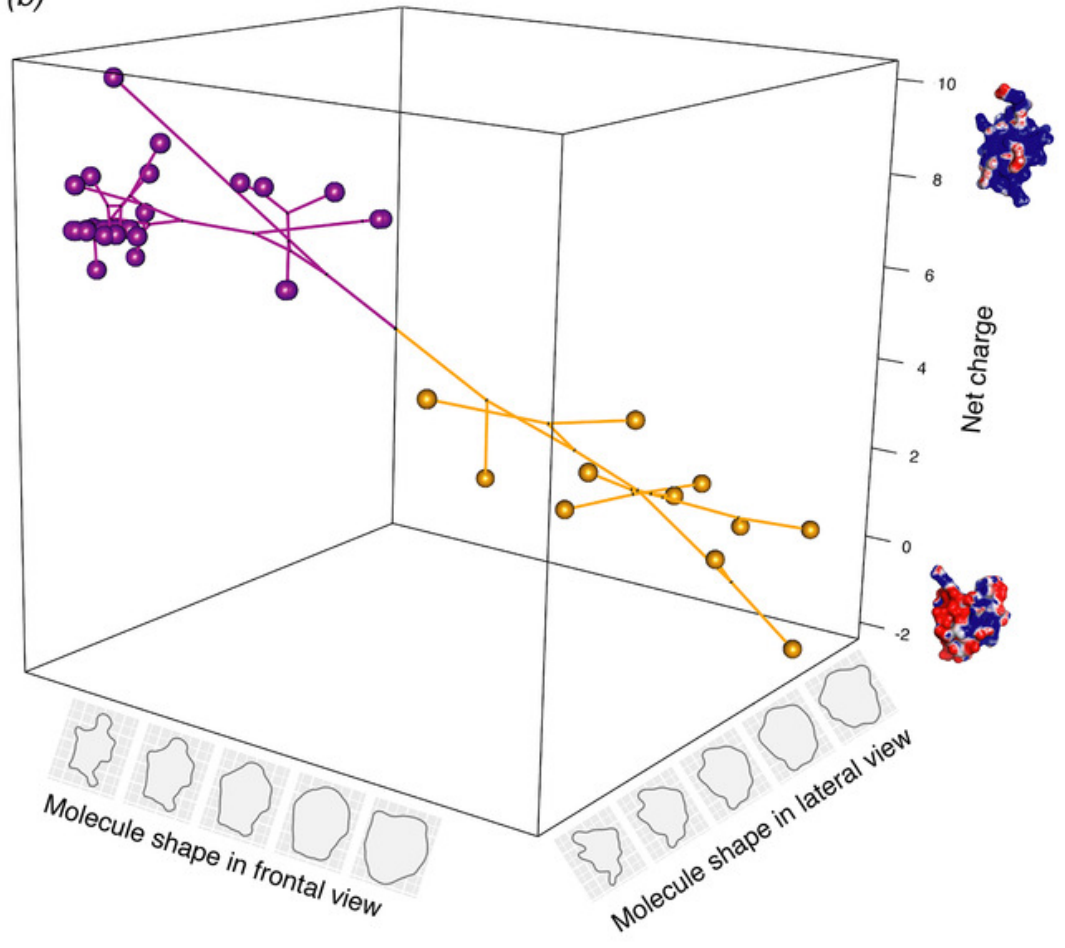

(d)

Buthoidea Chaeriloidea Pseudochactoidea Imperacalcin Peer] reviewing PD
-
- GCNRLNKKC - NSDGDCCRYGERCI STGV - NYY'CRPDFGP TECQ SKGT YCRNPLV SNCCP - GLWCL - . - . HSFCI E - . KECRKQSQHC - PRGFPCCP - GLVCIPV- - - RQICHTHI- T GDCLPHLKRC - - KADNDCC - GKKCKRRGTNAEKRCR - - - - 


\section{Figure 7}

Evolutionary shifts in optimum morphology and chemical properties of the toxins

Shifts in ICK peptide morphology. IIou and pBIC provided support for eight evolutionary shifts in optimum morphology under an Ornstein-Uhlenbeck (OU) process. Edges with a major morphological evolutionary shift are annotated with a star and bootstrap support (a). Bar graphs showing the seven traits combined: PC1 $(b, d)$ and PC2 $(c, e)$ from the frontal $(b, c)$ and lateral $(d, e)$ shape data; molecular weight $(f)$, molecular volume $(g)$, and net charge $(h)$.

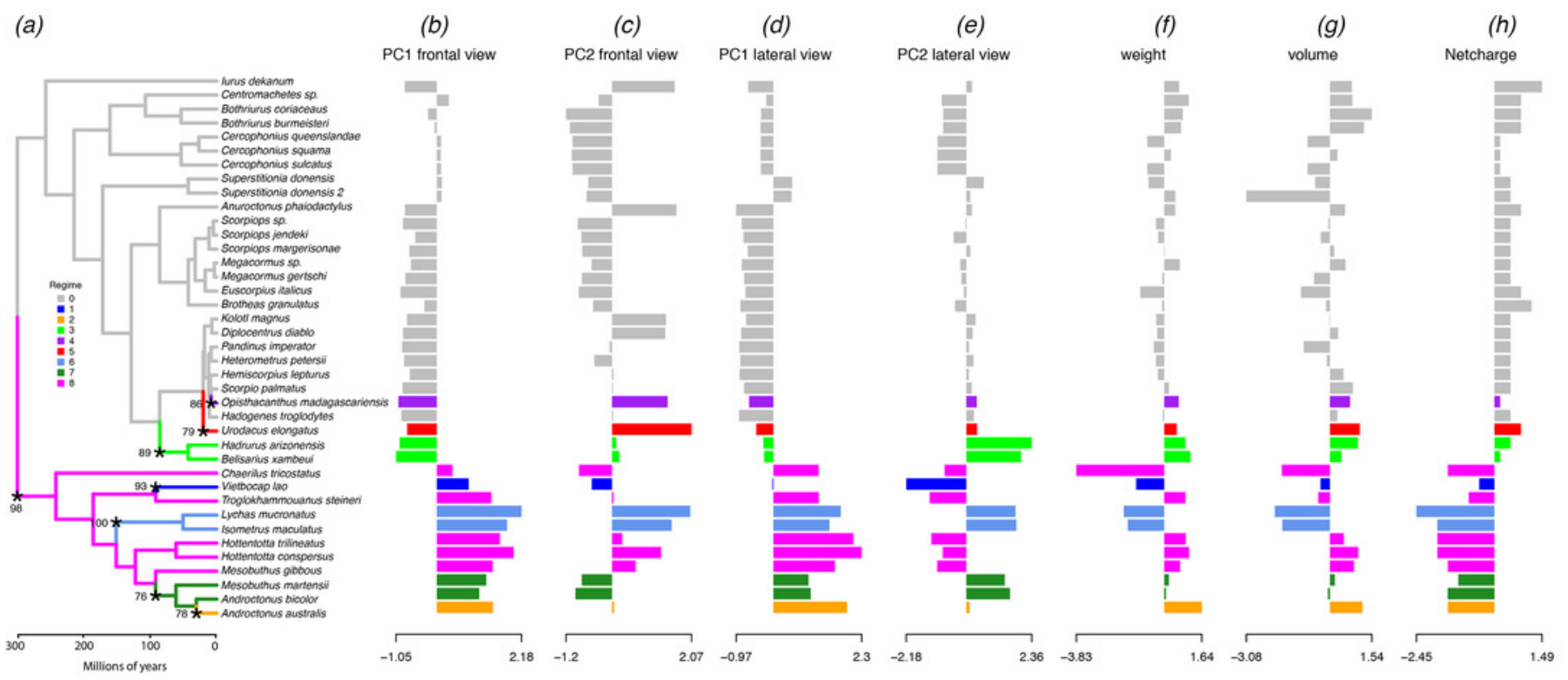

